RIMS-1742

Conditional results on the birational section conjecture over small number fields

By

Yuichiro HOSHI

February 2012

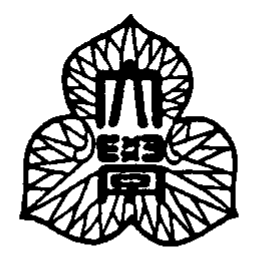

京都大学 数理解析研究所

RESEARCH INSTITUTE FOR MATHEMATICAL SCIENCES

KYOTO UNIVERSITY, Kyoto, Japan 


\title{
CONDITIONAL RESULTS ON THE BIRATIONAL SECTION CONJECTURE OVER SMALL NUMBER FIELDS
}

\author{
YUICHIRO HOSHI
}

FEBRUARY 2012

\begin{abstract}
In the present paper, we give necessary and sufficient conditions for a birational Galois section of a projective smooth curve over either the field of rational numbers or an imaginary quadratic field to be geometric. As a consequence, we prove that, over such a small number field, to prove the birational section conjecture for projective smooth curves, it suffices to verify that, roughly speaking, for any birational Galois section of the projective line, the local points associated to the birational Galois section avoid distinct three rational points, and, moreover, a certain Galois representation determined by the birational Galois section is unramified at all but finitely many primes. Moreover, as another consequence, we obtain some examples of projective smooth curves for which any prosolvable birational Galois section is geometric.
\end{abstract}

\section{CONTENTS}

Introduction 1

0. Notations and Conventions 7

1. Birational Galois sections and their geometricity 8

2. Local geometricity of birational Galois sections 14

3. Galois sections of tori that locally arise from points 21

4. Conditional results on the birational section conjecture 25

Appendix A. Ramification of Galois sections 36

References 44

\section{INTRODUCTION}

Let $k$ be a field of characteristic $0, \bar{k}$ an algebraic closure of $k$, and $X$ a projective smooth geometrically connected curve over $k$. Write $G_{k} \stackrel{\text { def }}{=}$ $\mathrm{Gal}(\bar{k} / k)$ for the absolute Galois group of $k$ determined by the fixed algebraic closure $\bar{k}$ of $k$. Now we have a natural surjection

$$
\pi_{1}(X) \rightarrow G_{k}
$$


from the étale fundamental group $\pi_{1}(X)$ of $X$ to $G_{k}$ induced by the structure morphism of $X$. Then Grothendieck's section conjecture may be stated as follows: If $k$ is finitely generated over the field of rational numbers, and $X$ is of genus $\geq 2$, then any section of this surjection $\pi_{1}(X) \rightarrow G_{k}$ arises from a $k$-rational point of $X$, i.e., the image of any section of this surjection coincides with, or, equivalently, is contained in, a decomposition subgroup of $\pi_{1}(X)$ associated to a $k$ rational point of $X$. In the present paper, we discuss the birational version of this conjecture, i.e., the birational section conjecture. Denote by $k(X)$ the function field of $X$. Fix an algebraic closure $\overline{k(X)}$ of $k(X)$ containing $\bar{k}$ and write $G_{k(X)} \stackrel{\text { def }}{=} \operatorname{Gal}(\overline{k(X)} / k(X))$. Then the natural inclusions $k \hookrightarrow k(X), \bar{k} \hookrightarrow \overline{k(X)}$ determine a surjection

$$
G_{k(X)} \rightarrow G_{k},
$$

which factors through the above surjection $\pi_{1}(X) \rightarrow G_{k}$. We shall refer to a section of this surjection $G_{k(X)} \rightarrow G_{k}$ as a [pro-Primes] birational Galois section of $X / k$ [cf. Definition 1.2]. In the present paper, we discuss the geometricity of birational Galois sections.

Let $x$ be a closed point of $X$ and $D_{x} \subseteq G_{k(X)}$ a decomposition subgroup of $G_{k(X)}$ associated to $x$. Then, as is well-known, the image of the composite $D_{x} \hookrightarrow G_{k(X)} \rightarrow G_{k}$ coincides with the open subgroup $G_{k(x)} \subseteq G_{k}$ of $G_{k}$ corresponding to the residue field $k(x)$ of $X$ at $x$, and, moreover, the resulting surjection $D_{x} \rightarrow G_{k(x)}$ admits a [not necessarily unique] section. In particular, if, moreover, $k(x)=x$, i.e., $x \in X(k)$, then the closed subgroup $D_{x} \subseteq G_{k(X)}$ of $G_{k(X)}$ contains the image of a [not necessarily unique] birational Galois section of $X / k$. We shall say that a birational Galois section of $X / k$ is geometric if its image is contained in a decomposition subgroup of $G_{k(X)}$ associated to a [necessarily $k$-rational] closed point of $X$ [cf. Definition 1.3].

The birational section conjecture over local fields has been solved affirmatively. In [8], Koenigsmann proved that if $k$ is either a $p$-adic local field for some prime number $p$ [i.e., a finite extension of the $p$ adic completion of the field of rational numbers] or the field of real numbers, then any birational Galois section of $X / k$ is geometric [cf. [8] Proposition 2.4, (2)]. Moreover, in [12], Pop obtained a result concerning birational Galois sections over $p$-adic local fields [cf. [12], Theorem A], which leads naturally to a proof of the geometrically pro$p$ version of Koenigsmann's result over $p$-adic local fields [cf. Proposition 1.7]. In [16], Wickelgren proved a strong version of the birational section conjecture over the field of real numbers [cf. [16], Corollary 1.2].

In the rest of the present introduction, we discuss the geometricity of birational Galois sections over number fields; suppose that $k$ is a number field [i.e., a finite extension of the field of rational numbers].

First, let us recall that, in [1], Esnault and Wittenberg proved that if the Shafarevich-Tate group of the Jacobian variety of $X$ over $k$ is 
finite, then the existence of a birational Galois section of $X / k$ implies the existence of a divisor of degree 1 on $X$; more precisely, the existence of a section of the natural surjection $G_{k(X)} /\left[G_{\bar{k} \cdot k(X)}, G_{\bar{k} \cdot k(X)}\right] \rightarrow G_{k}$, where we write $G_{\bar{k} \cdot k(X)} \stackrel{\text { def }}{=} \operatorname{Gal}(\overline{k(X)} / \bar{k} \cdot k(X))$ and $\left[G_{\bar{k} \cdot k(X)}, G_{\bar{k} \cdot k(X)}\right]$ for the closure of the commutator subgroup of $G_{\bar{k} \cdot k(X)}$, is equivalent to the existence of a divisor of degree 1 on $X$ [cf. [1], Theorem 2.1]. Next, let us recall that, in [4], Harari and Stix proved, as a consequence of results obtained by Stoll in [14], that if there exist an abelian variety $A$ over $k$ and a nonconstant morphism $X \rightarrow A$ over $k$ such that both the Mordell-Weil group and the Shafarevich-Tate group of $A / k$ are finite, then any birational Galois section of $X / k$ is geometric [cf. [4], Theorem 17]. This result of Harari and Stix gives us some examples of $X / k$ for which any birational Galois section is geometric [cf. [4], Remark 18, (1)].

To state our main results, let us discuss local points associated to a birational Galois section. Write $\mathfrak{P}_{k}^{f}$ for the set of nonarchimedean primes of $k$. For each $\mathfrak{p} \in \mathfrak{P}_{k}^{f}$, fix an algebraic closure $\bar{k}_{\mathfrak{p}}$ of the $\mathfrak{p}$-adic completion $k_{\mathfrak{p}}$ of $k$ containing $\bar{k}$ and write $G_{\mathfrak{p}} \stackrel{\text { def }}{=} \operatorname{Gal}\left(\bar{k}_{\mathfrak{p}} / k_{\mathfrak{p}}\right) \subseteq G_{k}$. Finally, write $\mathbb{A}_{k}^{f} \subseteq \prod_{\mathfrak{p} \in \mathfrak{P}_{k}^{f}} k_{\mathfrak{p}}$ for the finite part of the adele ring of $k$, i.e., the subring of $\prod_{\mathfrak{p} \in \mathfrak{P}_{k}^{f}} k_{\mathfrak{p}}$ consisting of elements $\left(a_{\mathfrak{p}}\right)_{\mathfrak{p} \in \mathfrak{P}_{k}^{f}} \in \prod_{\mathfrak{p} \in \mathfrak{P}_{k}^{f}} k_{\mathfrak{p}}$ such that $a_{\mathfrak{p}}$ is contained in the ring of integers of $k_{\mathfrak{p}}$ for all but finitely many $\mathfrak{p} \in \mathfrak{P}_{k}^{f}$. Then it follows from a result obtained in [8], as well as [12], that, for each $\mathfrak{p} \in \mathfrak{P}_{k}^{f}$, a birational Galois section $s$ of $X / k$ uniquely determines a $k_{\mathfrak{p}}$-valued point $x_{\mathfrak{p}}$ of $X$ such that, for any open subscheme $U \subseteq X$ of $X$, the image of the homomorphism $G_{\mathfrak{p}} \rightarrow \pi_{1}\left(U \otimes_{k} k_{\mathfrak{p}}\right)$ naturally determined by the isomorphism $\pi_{1}\left(U \otimes_{k} k_{\mathfrak{p}}\right) \stackrel{\sim}{\rightarrow} \pi_{1}(U) \times_{G_{k}} G_{\mathfrak{p}}$ and the composite $G_{\mathfrak{p}} \hookrightarrow$ $G_{k} \stackrel{s}{\rightarrow} G_{k(X)} \rightarrow \pi_{1}(U)$ is contained in a decomposition subgroup of $\pi_{1}\left(U \otimes_{k} k_{\mathfrak{p}}\right)$ associated to $x_{\mathfrak{p}}$ [cf. Proposition 2.9]; we shall refer to the $k_{\mathfrak{p}}$-valued point $x_{\mathfrak{p}}$ as the $k_{\mathfrak{p}}$-valued point of $X$ associated to $s$ [cf. Definition 2.6]. In particular, [since $X$ is projective over $k$ ] the birational Galois section $s$ uniquely determines an $\mathbb{A}_{k}^{f}$-valued point $x_{\mathbb{A}} \stackrel{\text { def }}{=}\left(x_{\mathfrak{p}}\right)_{\mathfrak{p} \in \mathfrak{P}_{k}^{f}} \in X\left(\mathbb{A}_{k}^{f}\right) \subseteq \prod_{\mathfrak{p} \in \mathfrak{P}_{k}^{f}} X\left(k_{\mathfrak{p}}\right)$ of $X$; we shall refer to the $\mathbb{A}_{k}^{f}$-valued point $x_{\mathbb{A}}$ as the $\mathbb{A}_{k}^{f}$-valued point of $X$ associated to $s$ [cf. Definition 2.6]. Note that if the birational Galois section $s$ is geometric, then there exists a [necessarily unique] $k$-rational point $x \in X(k)$ of $X$ such that, for each $\mathfrak{p} \in \mathfrak{P}_{k}^{f}$, the $k_{\mathfrak{p}}$-valued point of $X$ determined by $x$ is the $k_{\mathfrak{p}}$-valued point of $X$ associated to $s$ [cf. Remark 2.6.1].

Now let us recall a conditional result on the section conjecture over number fields. It seems to the author that [at least, a similar result to] the following result is likely to be well-known to expert; since, however, the result could not be found in the literature, the author 
decided to give a proof [cf. Theorem 4.1 in the case where $\mathcal{C}$ consists of all finite groups, and $s$ is a pro-C birational Galois section].

Theorem A. Let $k$ be a number field, $X$ a projective smooth geometrically connected curve over $k$, and $s$ a [pro-Primes] birational Galois section of $X / k[c f$. Definition 1.2]. Then the following conditions are equivalent:

(1) $s$ is geometric [cf. Definition 1.3].

(2) There exist a subset $T \subseteq \mathfrak{P}_{k}^{f}$ of $\mathfrak{P}_{k}^{f}$ of density 0 and a closed subscheme $Z \subseteq X$ of $X$ which is finite over $k$ such that, for each $\mathfrak{p} \in \mathfrak{P}_{k}^{f} \backslash T$, [the image of] the $k_{\mathfrak{p}}$-valued point of $X$ associated to $s$ is contained in $Z \subseteq X$.

The following result is our second main result, which gives necessary and sufficient conditions for a birational Galois section of a projective smooth geometrically connected curve over a small number field, i.e., either the field of rational numbers or an imaginary quadratic field, to be geometric [cf. Theorem 4.5 in the case where $\mathcal{C}$ consists of all finite groups].

Theorem B. Let $k$ be either the field of rational numbers or an imaginary quadratic field, $X$ a projective smooth geometrically connected curve over $k$, and $s$ a $[$ pro-Primes $]$ birational Galois section of $X / k[c f$. Definition 1.2]. Then the following conditions are equivalent:

(1) $s$ is geometric [cf. Definition 1.3].

(2) The following two conditions are satisfied:

(2-i) There exists a finite morphism $\phi: X \rightarrow \mathbb{P}_{k}^{1}$ over $k$ such that, for each $\mathfrak{p} \in \mathfrak{P}_{k}^{f}$, the composite

$$
\text { Spec } k_{\mathfrak{p}} \stackrel{x_{\mathfrak{p}}}{\longrightarrow} X \stackrel{\phi}{\longrightarrow} \mathbb{P}_{k}^{1}
$$

determines a $k_{\mathfrak{p}}$-valued point of $\mathbb{P}_{k}^{1} \backslash\{0,1, \infty\}$.

(2-ii) For each open subscheme $U \subseteq X$ of $X$ which is a hyperbolic curve over $k$ [where we refer to the discussion entitled "Curves" in $\S 0$ concerning the term "hyperbolic curve"], there exists a prime number $l_{U}$ such that the pro- $l_{U}$ Galois section of $U / k$ [cf. Definition 1.2] naturally determined by $s$ is either cuspidal $[c f$. Definition 4.3, (i)] or unramified almost everywhere [cf. Definition 4.3, (ii)].

(3) There exists a finite morphism $\phi: X \rightarrow \mathbb{P}_{k}^{1}$ over $k$ such that the composite

$$
\operatorname{Spec} \mathbb{A}_{k}^{f} \stackrel{x_{\mathbb{A}}}{\longrightarrow} X \stackrel{\phi}{\longrightarrow} \mathbb{P}_{k}^{1}
$$

determines an $\mathbb{A}_{k}^{f}$-valued point of $\mathbb{P}_{k}^{1} \backslash\{0,1, \infty\} \subseteq \mathbb{P}_{k}^{1}$.

(4) There exist a finite subset $T \subseteq \mathfrak{P}_{k}^{f}$ of $\mathfrak{P}_{k}^{f}$ and a closed subscheme $Z \subseteq X$ of $X$ which is finite over $k$ such that, for each $\mathfrak{p} \in \mathfrak{P}_{k}^{f} \backslash T$, 
[the image of] the $k_{\mathfrak{p}}$-valued point $x_{\mathfrak{p}}$ of $X$ associated to $s$ is contained in $Z \subseteq X$.

Note that Theorem B is a result without any assumption on the finiteness of a Shafarevich-Tate group. Next, let us observe that the equivalence $(1) \Leftrightarrow(3)$ of Theorem B may be regarded as a tripod analogue of the result due to Harari and Stix discussed above, i.e., [4], Theorem 17. The condition that $k$ is either the field of rational numbers or an imaginary quadratic field [i.e., the assumption that the group of units of the ring of integers of $k$ is finite] in the statement of Theorem B may be regarded as an analogue of the finiteness condition on the Mordell-Weil group in the statement of [4], Theorem 17; on the other hand, since any abelian variety is proper, in the case of [4], Theorem 17, the condition corresponding to our condition that the birational Galois section determines [not only a $\left(\prod_{\mathfrak{p} \in \mathfrak{P}_{k}^{f}} k_{\mathfrak{p}}\right)$-valued point but also] an $\mathbb{A}_{k}^{f}$-valued point of the tripod $\mathbb{P}_{k}^{1} \backslash\{0,1, \infty\}$ in Theorem B is automatically satisfied. Finally, in this case [i.e., in the case where $\mathcal{C}$ consists of all finite groups], the equivalence $(1) \Leftrightarrow(4)$ of Theorem B is a consequence of Theorem A.

As a corollary of Theorem B, we prove the following result [cf. Corollary 4.6].

Theorem C. Let $k$ be either the field of rational numbers or an imaginary quadratic field. Then the following assertions are equivalent:

(1) Any [pro-Primes] birational Galois section [cf. Definition 1.2] of any projective smooth geometrically connected curve over $k$ is geometric [cf. Definition 1.3].

(2) Any [pro-Primes] birational Galois section of $\mathbb{P}_{k}^{1} / k$ is geometric.

(3) Any [pro-Primes] birational Galois section s of $\mathbb{P}_{k}^{1} / k$ satisfies the following two conditions:

(3-i) There exist three distinct elements $a, b, c \in \mathbb{P}_{k}^{1}(k)$ of $\mathbb{P}_{k}^{1}(k)$ such that, for any nonarchimedean prime $\mathfrak{p}$ of $k$, the $k_{\mathfrak{p}}$-valued point of $\mathbb{P}_{k}^{1}$ associated to $s$ is $\notin\{a, b, c\} \subseteq\left(\mathbb{P}_{k}^{1}(k) \subseteq\right) \mathbb{P}_{k}^{1}\left(k_{\mathfrak{p}}\right)$.

(3-ii) There exists a prime number $l$ such that the pro-l Galois section of $\mathbb{P}_{k}^{1} \backslash\{0,1, \infty\}$ [cf. Definition 1.2] naturally determined by $s$ is either cuspidal [cf. Definition 4.3, (i)] or unramified almost everywhere $[c f$. Definition 4.3 , (ii)].

(4) Any [pro-Primes] birational Galois section $s$ of $\mathbb{P}_{k}^{1} / k$ satisfies the following two conditions:

(4-i) There exist three distinct elements $a, b, c \in \mathbb{P}_{k}^{1}(k)$ of $\mathbb{P}_{k}^{1}(k)$ such that, for any nonarchimedean prime $\mathfrak{p}$ of $k$, the $k_{\mathfrak{p}}$-valued point of $\mathbb{P}_{k}^{1}$ associated to $s$ is $\notin\{a, b, c\} \subseteq\left(\mathbb{P}_{k}^{1}(k) \subseteq\right) \mathbb{P}_{k}^{1}\left(k_{\mathfrak{p}}\right)$.

(4-ii) Write $s^{\mathbb{P}}$ for the pro-Primes Galois section of $\mathbb{P}_{k}^{1} \backslash\{0,1, \infty\}[c f$. Definition 1.2] naturally determined by s. Then it holds either that $s^{\mathbb{P}}$ is cuspidal $[c f$. Definition 4.3, (i)], or that there exists 
a prime number l such that the l-adic Galois representation

$$
G_{k} \stackrel{s^{\mathbb{P}}}{\longrightarrow} \pi_{1}\left(\mathbb{P}_{k}^{1} \backslash\{0,1, \infty\}\right) \longrightarrow \mathrm{GL}_{2}\left(\mathbb{Z}_{l}\right)
$$

- where the second arrow $\pi_{1}\left(\mathbb{P}_{k}^{1} \backslash\{0,1, \infty\}\right) \rightarrow \mathrm{GL}_{2}\left(\mathbb{Z}_{l}\right)$ is the l-adic representation of $\pi_{1}\left(\mathbb{P}_{k}^{1} \backslash\{0,1, \infty\}\right)$ determined by the Legendre family of elliptic curves over $\mathbb{P}_{k}^{1} \backslash\{0,1, \infty\}$, i.e., the elliptic curve over $\mathbb{P}_{k}^{1} \backslash\{0,1, \infty\}=\operatorname{Spec} k\left[u^{ \pm 1},(1-\right.$ $u)^{-1}$ determined by the equation " $y^{2}=x(x-1)(x-u)^{\prime \prime}-$ is unramified at all but finitely many primes of $k$.

As a consequence [cf. the equivalences (1) $\Leftrightarrow(3)$ and $(1) \Leftrightarrow(4)$ of Theorem C], for a number field $k$ which is either the field of rational numbers or an imaginary quadratic field, to prove the birational section conjecture over $k$ [i.e., assertion (1) of Theorem C], it suffices to verify that, roughly speaking, for any birational Galois section of the projective line over $k$, the local points associated to the birational Galois section avoid distinct three rational points [cf. conditions (3-i), (4-i)], and, moreover, a certain Galois representation determined by the birational Galois section is unramified at all but finitely many primes [cf. conditions (3-ii), (4-ii)]. However, it is not clear to the author at the time of writing whether or not these are always satisfied.

Finally, let us observe that Theorem 4.1 leads naturally to the following generalization of the above result obtained by Harari and Stix [cf. Corollary 4.2 in the case where $s$ is a pro-C birational Galois section]. Note that if $k$ is either the field of rational numbers or an imaginary quadratic field, then the following result may also be derived from Theorem 4.5 [cf. Remark 4.5.1, (iv)].

Theorem D. Let $\mathcal{C}$ be a full formation that contains all finite solvable groups, $k$ a number field, and $X$ a projective smooth geometrically connected curve over $k$. Suppose that there exist an abelian variety $A$ over $k$ and $a$ nonconstant morphism $X \rightarrow A$ over $k$ such that both the Mordell-Weil group and the Shafarevich-Tate group of $A / k$ are finite. Then any pro-C birational Galois section of $X / k[c f$. Definition 1.2] is geometric [cf. Definition 1.3].

As in the case of the result of Harari and Stix, this result gives us some examples of $X / k$ for which any prosolvable birational Galois section is geometric [cf. Remark 4.2.1].

\section{ACKNOWLEDGEMENTS}

The author would like to thank Akio Tamagawa, Takahiro Tsushima, and Seidai Yasuda for their helpful comments and, especially, discussions concerning $§ 3$. This research was supported by Grant-in-Aid for Young Scientists (B), No. 22740012, Japan Society for the Promotion of Science. 


\section{NOTATIONS AND CONVENTIONS}

Numbers: The notation Primes will be used to denote the set of all prime numbers. The notation $\mathbb{Z}$ will be used to denote the ring of rational integers. If $\Sigma \subseteq \mathfrak{P r i m e s}$, then we shall refer to a nonzero integer whose prime divisors are $\in \Sigma$ as a $\Sigma$-integer, and we shall write $\widehat{\mathbb{Z}}^{\Sigma}$ for the pro- $\Sigma$ completion of $\mathbb{Z}$, i.e., $\widehat{\mathbb{Z}}^{\Sigma} \stackrel{\text { def }}{=} \lim \mathbb{Z} / n \mathbb{Z}$, where the projective limit is over all positive $\Sigma$-integers $n$. We shall refer to a finite (respectively, finitely generated) extension of the field of rational numbers as a number field (respectively, finitely generated field of characteristic 0 ). If $p \in \mathfrak{P r i m e s}$, then the notation $\mathbb{Z}_{p}$ will be used to denote the $p$-adic completion of $\mathbb{Z}$, and we shall refer to a finite extension of the $p$-adic completion of the field of rational numbers as a $p$-adic local field.

Profinite groups: Let $G$ be a profinite group and $H \subseteq G$ a closed subgroup of $G$. Then we shall denote by $Z_{G}(H), N_{G}(H), Z_{G}^{\text {loc }}(H)$ the centralizer, normalizer, local centralizer of $H$ in $G$, respectively, i.e.,

$$
\begin{gathered}
Z_{G}(H) \stackrel{\text { def }}{=}\left\{g \in G \mid g h g^{-1}=h \text { for any } h \in H\right\} \\
N_{G}(H) \stackrel{\text { def }}{=}\left\{g \in G \mid g \cdot H \cdot g^{-1}=H\right\} \\
Z_{G}^{\text {loc }}(H) \stackrel{\text { def }}{=} \underset{U}{\lim } Z_{G}(U)
\end{gathered}
$$

- where the injective limit is over all open subgroups $U \subseteq H$ of $H$. We shall refer to $Z(G) \stackrel{\text { def }}{=} Z_{G}(G), Z^{\text {loc }}(G) \stackrel{\text { def }}{=} Z_{G}^{\text {loc }}(G)$ as the center, local center of $G$, respectively. We shall say that $G$ is center-free, slim if $Z(G)=\{1\}, Z^{\mathrm{loc}}(G)=\{1\}$, respectively.

Let $\Sigma \subseteq \mathfrak{P r i m e s}$ be a nonempty subset of $\mathfrak{P r i m e s}$ [where we refer to the discussion entitled "Numbers" concerning the set $\mathfrak{P r i m e s}]$. Then we shall say that a finite group $G$ is $\Sigma$-group if the cardinality of $G$ is a $\Sigma$-integer [where we refer to the discussion entitled "Numbers" concerning the term " $\Sigma$-integer"].

Let $\mathcal{C}$ be a full formation [i.e., a family of finite groups that is closed under taking quotients, subgroups, and extensions]. We shall say that a finite group is a $\mathcal{C}$-group if [a finite group which is isomorphic to] the finite group is contained in $\mathcal{C}$. We shall say that a profinite group is a pro- $\mathcal{C}$ group if every finite quotient of the profinite group is a $\mathcal{C}$-group. We shall write $\Sigma(\mathcal{C}) \subseteq \mathfrak{P r i m e s}$ for the set of prime numbers $p \in \mathfrak{P r i m e s}$ such that $\mathbb{Z} / p \mathbb{Z}$ is a $\mathcal{C}$-group. Here, we note that one verifies easily that $\Sigma(\mathcal{C})=\mathfrak{P r i m e s}$ if and only if $\mathcal{C}$ contains all finite solvable groups. If $\mathcal{C}$ consists of all $\Sigma$-groups for some nonempty subset $\Sigma \subseteq$ Primes, then we shall refer to a pro-C group as a pro- $\Sigma$ group. 
Let $G$ be a profinite group. Then we shall write $G^{\text {ab }}$ for the abelianization of $G$, i.e., the quotient $G /[G, G]$ of $G$ by the closure $[G, G]$ of the commutator subgroup of $G$.

Let $G$ be a profinite group. Then we shall write $\operatorname{Aut}(G)$ for the group of [continuous] automorphisms of $G, \operatorname{Inn}(G) \subseteq \operatorname{Aut}(G)$ for the group of inner automorphisms of $G$, and

$$
\operatorname{Out}(G) \stackrel{\text { def }}{=} \operatorname{Aut}(G) / \operatorname{Inn}(G) \text {. }
$$

If, moreover, $G$ is topologically finitely generated, then one verifies easily that the topology of $G$ admits a basis of characteristic open subgroups, which thus induces a profinite topology on the group $\operatorname{Aut}(G)$, hence also a profinite topology on the group $\operatorname{Out}(G)$.

Curves: Let $S$ be a scheme and $X$ a scheme over $S$. Then we shall say that $X$ is a smooth curve over $S$ if there exist a scheme $X^{\mathrm{cpt}}$ which is smooth, proper, geometrically connected, and of relative dimension 1 over $S$ and a closed subscheme $D \subseteq X^{\mathrm{cpt}}$ of $X^{\mathrm{cpt}}$ which is finite and étale over $S$ such that the complement $X^{\mathrm{cpt}} \backslash D$ of $D$ in $X^{\mathrm{cpt}}$ is isomorphic to $X$ over $S$. Note that, as is well-known, if $X$ is a smooth curve over [the spectrum of] a field $k$, then the pair " $\left(X^{\mathrm{cpt}}, D\right)$ " is uniquely determined up to canonical isomorphism over $k$; we shall refer to $X^{\mathrm{cpt}}$ as the smooth compactification of $X$ over $k$ and to a geometric point of $X^{\text {cpt }}$ whose image lies on $D$ as a cusp of $X$.

Let $S$ be a scheme. Then we shall say that a smooth curve $X$ over $S$ is a hyperbolic curve (respectively, tripod) over $S$ if there exist a pair $\left(X^{\mathrm{cpt}}, D\right)$ satisfying the condition in the above definition of the term "smooth curve" and a pair $(g, r)$ of nonnegative integers such that $2 g-2+r>0$ (respectively, $(g, r)=(0,3)$ ), any geometric fiber of $X^{\mathrm{cpt}} \rightarrow S$ is [a necessarily smooth, proper, and connected curve] of genus $g$, and the degree of $D \subseteq X^{\mathrm{cpt}}$ over $S$ is $r$.

Let $S$ be a scheme, $U \subseteq S$ an open subscheme of $S$, and $X$ a hyperbolic curve over $U$. Then we shall say that $X$ admits good reduction over $S$ if there exists a hyperbolic curve $X_{S}$ over $S$ such that $X_{S} \times{ }_{S} U$ is isomorphic to $X$ over $U$.

\section{BirAtionAl GALOIS SECTIONS AND THEIR GEOMETRICITY}

In the present $\$ 1$, we discuss the notion of a birational Galois section. In the present $\S 1$, let $\mathcal{C}$ be a full formation, $k$ a field of characteristic 0 , and $\bar{k}$ an algebraic closure of $k$. For a finite extension $k^{\prime}(\subseteq \bar{k})$ of $k$, write $G_{k^{\prime}} \stackrel{\text { def }}{=} \operatorname{Gal}\left(\bar{k} / k^{\prime}\right)$.

Definition 1.1. Let $X$ be a quasi-compact scheme which is geometrically integral over $k$.

(i) We shall write 
for the function field of $X$.

(ii) We shall write

$$
\Delta_{X / k}^{\mathcal{C}}
$$

for the pro-C geometric fundamental group of $X$, i.e., the maximal pro- $\mathcal{C}$ quotient of $\pi_{1}\left(X \otimes_{k} \bar{k}\right)$, and

$$
\Pi_{X / k}^{\mathcal{C}}
$$

for the geometrically pro-C fundamental group of $X$, i.e., the quotient of $\pi_{1}(X)$ by the kernel of the natural surjection $\pi_{1}\left(X \otimes_{k}\right.$ $\bar{k}) \rightarrow \Delta_{X / k}^{\mathcal{C}}$. If $X$ is the spectrum of a ring $R$, then we shall write

$$
\Delta_{R / k}^{\mathcal{C}} \stackrel{\text { def }}{=} \Delta_{X / k}^{\mathcal{C}} ; \Pi_{R / k}^{\mathcal{C}} \stackrel{\text { def }}{=} \Pi_{X / k}^{\mathcal{C}} .
$$

Thus, we have a commutative diagram of profinite groups

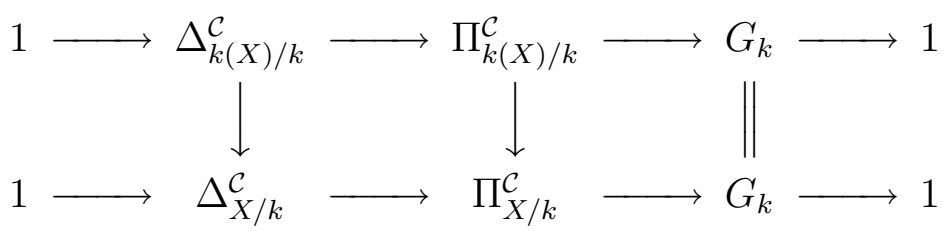

- where the horizontal sequences are exact [cf. [3], Exposé IX, Théorème 6.1].

If $\mathcal{C}$ consists of all $\Sigma$-groups [where we refer to the discussion entitled "Profinite groups" in $\S 0$ concerning the term " $\Sigma$ group"] for some nonempty subset $\Sigma \subseteq$ Primes [where we refer to the discussion entitled "Numbers" in $\S 0$ concerning the set $\mathfrak{P r i m} \mathfrak{r}$ ], then we shall write

$$
\Delta_{X / k}^{\Sigma} \stackrel{\text { def }}{=} \Delta_{X / k}^{\mathcal{C}} ; \Pi_{X / k}^{\Sigma} \stackrel{\text { def }}{=} \Pi_{X / k}^{\mathcal{C}} .
$$

Definition 1.2. Let $X$ be a quasi-compact scheme which is geometrically integral over $k$. Then we shall refer to a section of the upper (respectively, lower) exact sequence of the commutative diagram of Definition 1.1, (ii), as a pro-C $\mathcal{C}$ birational Galois section (respectively, pro$\mathcal{C}$ Galois section) of $X / k$. The $\Delta_{k(X) / k}^{\mathcal{C}}$-conjugacy (respectively, $\Delta_{X / k}^{\mathcal{C}}$ conjugacy) class of a pro- $\mathcal{C}$ birational Galois section (respectively, pro- $\mathcal{C}$ Galois section) of $X / k$ as the conjugacy class of the pro- $\mathcal{C}$ birational Galois section (respectively, pro- $\mathcal{C}$ Galois section).

If $\mathcal{C}$ consists of all $\Sigma$-groups for some nonempty subset $\Sigma \subseteq \mathfrak{P r i m e s}$, then we shall refer to a pro- $\mathcal{C}$ birational Galois section (respectively, pro-C Galois section) of $X / k$ as a pro- $\Sigma$ birational Galois section (respectively, pro- $\Sigma$ Galois section) of $X / k$.

Definition 1.3. Let $X$ be a smooth curve over $k$ [where we refer to the discussion entitled "Curves" in $\S 0$ concerning the term "smooth curve"] and $s$ a pro- $\mathcal{C}$ birational Galois section (respectively, pro- $\mathcal{C}$ Galois section) of $X / k$ [cf. Definition 1.2]. Then we shall say that $s$ is geometric if the image of $s$ is contained in a decomposition subgroup 
of $\Pi_{k(X) / k}^{\mathcal{C}}$ (respectively, $\Pi_{X / k}^{\mathcal{C}}$ ) associated to a [necessarily $k$-rational] closed point of the [uniquely determined] smooth compactification of $X$ over $k$.

Remark 1.3.1. Let $X$ be a scheme which is of finite type and geometrically integral over $k ; \Sigma \subseteq \mathfrak{P r i m e s}$ a nonempty subset of Primes. Then it is immediate that the notion of "pro- $\Sigma$ Galois section of $X / k$ " defined in Definition 1.2 coincides with the notion of "pro- $\Sigma$ Galois section of $X / k^{\prime \prime}$ defined in [6], Definition 1.1, (i). If, moreover, $X$ is a smooth curve over $k$, and $s$ is a pro- $\Sigma$ Galois section of $X / k$, then it is immediate that it holds that $s$ is geometric in the sense of Definition 1.3 if and only if $s$ is geometric in the sense of [6], Definition 1.1, (iii).

Remark 1.3.2. Let $X$ be a smooth curve over $k$. Then it follows immediately from the various definitions involved that the geometricity of a pro- $\mathcal{C}$ birational Galois section (respectively, pro- $\mathcal{C}$ Galois section) of $X / k$ depends only on its conjugacy class [cf. Definition 1.2].

Remark 1.3.3. Let $X, Y$ be smooth curves over $k$ and $Y \rightarrow X$ a dominant morphism over $k$, which thus determines a finite extension $k(X) \hookrightarrow k(Y)$ over $k$. If a pro-C $\mathcal{C}$ birational Galois section (respectively, pro- $\mathcal{C}$ Galois section) $s$ of $Y / k$ is geometric, then it follows immediately from the various definitions involved that the pro- $\mathcal{C}$ birational Galois section (respectively, pro-C Galois section) of $X / k$ determined by $s$ and the morphism $Y \rightarrow X$ [i.e., the pro- $\mathcal{C}$ birational Galois section (respectively, pro-C Galois section) of $X / k$ obtained as the composite of $s$ and the natural open homomorphism $\Pi_{k(Y) / k}^{\mathcal{C}} \rightarrow \Pi_{k(X) / k}^{\mathcal{C}}$ (respectively, $\Pi_{Y / k}^{\mathcal{C}} \rightarrow \Pi_{X / k}^{\mathcal{C}}$ ) induced by $\left.Y \rightarrow X\right]$ is geometric.

Remark 1.3.4. Let $X$ be a projective smooth curve over $k, U \subseteq X$ an open subscheme of $X$, and $s$ a pro- $\mathcal{C}$ birational Galois section of $X / k$. Then it follows immediately from the various definitions involved that if $s$ is geometric, then the pro- $\mathcal{C}$ Galois section of $U / k$ naturally determined by $s$ [i.e., the pro- $\mathcal{C}$ Galois section of $U / k$ obtained as the composite of $s$ and the natural surjection $\left.\Pi_{k(X) / k}^{\mathcal{C}} \rightarrow \Pi_{U / k}^{\mathcal{C}}\right]$ is geometric.

Lemma 1.4. Let $X$ be a hyperbolic curve over $k$ [where we refer to the discussion entitled "Curves" in $§ 0$ concerning the term "hyperbolic curve"] and $x, y$ closed points of the [uniquely determined] smooth compactification of $X$. Suppose that $k$ is generalized sub-p-adic [i.e., $k$ is isomorphic to a subfield of a finitely generated extension of the p-adic completion of the maximal unramified extension of the p-adic completion of the field of rational numbers - cf. [10], Definition 4.11] for some $p \in \Sigma(\mathcal{C})$ [where we refer to the discussion entitled "Profinite groups" in $§ 0$ concerning the set $\Sigma(\mathcal{C})$ ]. Then the following conditions are equivalent: 
(1) $x=y$.

(2) There exist respective decomposition subgroups $D_{x}, D_{y} \subseteq \Pi_{X / k}^{\mathcal{C}}$ of $\Pi_{X / k}^{\mathcal{C}}$ associated to $x, y$ such that the image of the composite

$$
D_{x} \cap D_{y} \hookrightarrow \Pi_{X / k}^{\mathcal{C}} \rightarrow G_{k}
$$

is open.

Proof. The implication $(1) \Rightarrow(2)$ is immediate. Next, we verify the implication $(2) \Rightarrow(1)$. Suppose that condition (2) is satisfied. Then it is immediate that, to verify the implication $(2) \Rightarrow(1)$, by replacing $\Pi_{X / k}^{\mathcal{C}}$ by an open subgroup of $\Pi_{X / k}^{\mathcal{C}}$, we may assume without loss of generality that $X$ is of genus $\geq 2$, and, moreover, the displayed composite of condition (2) is surjective, hence also that $x$ and $y$ are $k$-rational. Thus, to verify the implication $(2) \Rightarrow(1)$, by replacing $X$ by its smooth compactification, we may assume without loss of generality that $x, y \in X(k)$. Then, by considering the quotient $\Pi_{X / k}^{\mathcal{C}} \rightarrow \Pi_{X / k}^{\{p\}}$ of $\Pi_{X / k}^{\mathcal{C}}$, the implication $(2) \Rightarrow(1)$ follows immediately from [10], Theorem 4.12 [cf. also [10], Remark following Theorem 4.12], together with a similar argument to the argument used in the proof of [9], Theorem C. This completes the proof of the implication $(2) \Rightarrow(1)$, hence also of Lemma 1.4 .

The [equivalence $(1) \Leftrightarrow(3)$ of the] following result is a slight generalization of [8], Lemma 1.7, and follows essentially from the argument applied in the proof of [15], Proposition 2.8, (iv).

Lemma 1.5. Let $X$ be a smooth curve over $k$, s a pro-C birational Galois section of $X / k$ [cf. Definition 1.2], and $k^{\prime}(\subseteq \bar{k})$ a finite extension of $k$. Suppose that $k$ is either

(a) a finitely generated field of characteristic $\mathbf{0}$ [where we refer to the discussion entitled "Numbers" in $\S 0$ concerning the term "finitely generated field of characteristic 0"] or

(b) a p-adic local field [where we refer to the discussion entitled "Numbers" in $\S 0$ concerning the term " $p$-adic local field"] for some $p \in$ $\Sigma(\mathcal{C})$ [where we refer to the discussion entitled "Profinite groups" in $\S 0$ concerning the set $\Sigma(\mathcal{C})$ ].

Then the following conditions are equivalent:

(1) $s$ is geometric [cf. Definition 1.3].

(2) The pro-C birational Galois section $\left.s\right|_{G_{k^{\prime}}}$ of $X \otimes_{k} k^{\prime} / k^{\prime}$ determined by $s$ is geometric.

(3) For any open subgroup $H \subseteq \Pi_{k(X) / k}^{\mathcal{C}}$ of $\Pi_{k(X) / k}^{\mathcal{C}}$ containing the image of $s$, the [uniquely determined] smooth compactification of the normalization of $X$ in the finite extension of $k(X)$ corresponding to $H \subseteq \Pi_{k(X) / k}^{\mathcal{C}}$ admits a $k^{\prime}$-valued point. 
Proof. To verify Lemma 1.5 , by replacing $k^{\prime}$ by a finite extension of $k^{\prime}$, we may assume without loss of generality that $k^{\prime}$ is Galois over $k$. Moreover, to verify Lemma 1.5 , by replacing $\Pi_{k(X) / k}^{\mathcal{C}}$ by an open subgroup of $\Pi_{k(X) / k}^{\mathcal{C}}$ containing the image of $s$, we may assume without loss of generality that $X$ is of genus $\geq 2$.

Now the implication $(1) \Rightarrow(3)$ is immediate. Next, we verify the implication (3) $\Rightarrow(2)$. Suppose that condition (3) is satisfied. Write $\left\{H_{i}\right\}_{i \in I}$ for the projective system of open subgroups $H_{i} \subseteq \Pi_{k(X) / k}^{\mathcal{C}}$ of $\Pi_{k(X) / k}^{\mathcal{C}}$ containing the image of $s$. [Thus, we have that $\bigcap_{i} H_{i}=$ $\operatorname{Im}(s)$.] For each $i \in I$, write $X_{i}$ for the [uniquely determined] smooth compactification of the normalization of $X$ in the finite extension of $k(X)$ corresponding to $H_{i}$; thus, $\Pi_{k\left(X_{i}\right) / k}^{\mathcal{C}}=H_{i} \subseteq \Pi_{k(X) / k}^{\mathcal{C}}$, and we have a projective system $\left\{X_{i}\right\}_{i \in I}$ of proper hyperbolic curves over $k$ [with finite morphisms over $k$ ], hence also a projective system $\left\{X_{i}\left(k^{\prime}\right)\right\}_{i \in I}$ of sets. Now let us observe that if $k$ is a finitely generated field of characteristic 0 (respectively, $p$-adic local field), then it follows immediately from the Mordell conjecture that was proved by Faltings (respectively, from the consideration of models of the $X_{i}$ 's over the ring of integers of $k$ ) that, for each $i \in I, X_{i}\left(k^{\prime}\right)$ admits a compact topology with respect to which each morphism appearing in the projective system $\left\{X_{i}\left(k^{\prime}\right)\right\}_{i \in I}$ is continuous. Thus, since, for each $i \in I, X_{i}\left(k^{\prime}\right) \neq \emptyset$ by condition (3), it follows immediately that $\lim X_{i}\left(k^{\prime}\right) \neq \emptyset$. Let $x_{\infty} \in \lim X_{i}\left(k^{\prime}\right)$ be an element of $\lim X_{i}\left(k^{\prime}\right)$. Then

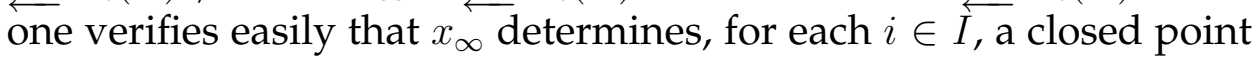
$x_{i}$ of $X_{i}$ which is defined over a subfield of $k^{\prime}$ and a decomposition subgroup $D_{i} \subseteq \Pi_{k\left(X_{i}\right) / k}^{\mathcal{C}}=H_{i}$ of $\Pi_{k\left(X_{i}\right) / k}^{\mathcal{C}}=H_{i}$ associated to $x_{i}$ which is compatible with each natural inclusion appearing in the projective system $\left\{H_{i}\right\}_{i \in I}$, and, moreover, the image of the composite

$$
\bigcap_{i} D_{i} \hookrightarrow \Pi_{k(X) / k}^{\mathcal{C}} \rightarrow G_{k}
$$

contains $G_{k^{\prime}} \subseteq G_{k}$. On the other hand, since $\bigcap_{i} D_{i} \subseteq \bigcap_{i} H_{i}=\operatorname{Im}(s)$, one verifies easily that $\operatorname{Im}\left(\left.s\right|_{G_{k^{\prime}}}\right) \subseteq \bigcap_{i} D_{i}$; in particular, $\operatorname{Im}\left(\left.s\right|_{G_{k^{\prime}}}\right)$ is contained in " $D_{i}$ " in the case where we take " $H_{i}$ " to be $\Pi_{k(X) / k}^{\mathcal{C}}$, i.e., $\left.s\right|_{G_{k^{\prime}}}$ is geometric. This completes the proof of the implication (3) $\Rightarrow$ (2).

Finally, we verify the implication $(2) \Rightarrow(1)$. Suppose that condition (2) is satisfied. Then, to verify the implication $(2) \Rightarrow(1)$, by the verified implication $(3) \Rightarrow(2)$ [in the case where we take " $k$ " to be $k]$, it suffices to verify that, for any open subgroup $H \subseteq \Pi_{k(X) / k}^{\mathcal{C}}$ of $\Pi_{k(X) / k}^{\mathcal{C}}$ containing the image of $s$, the [uniquely determined] smooth compactification $Y$ of the normalization of $X$ in the finite extension of $k(X)$ corresponding to $H \subseteq \Pi_{k(X) / k}^{\mathcal{C}}$ admits a $k$-rational point. 
Now it follows immediately from condition (2) that the pro-C birational Galois section of $Y \otimes_{k} k^{\prime} / k^{\prime}$ naturally determined by $s$ is geometric; thus, there exist a closed point $y$ of $Y$ which is defined over a subfield of $k^{\prime}$ and a decomposition subgroup $D_{y} \subseteq \Pi_{k(Y) / k}^{\mathcal{C}}=H$ of $\Pi_{k(Y) / k}^{\mathcal{C}}=H$ associated to $y$ such that $s\left(G_{k^{\prime}}\right) \subseteq D_{y}$. Let $g \in G_{k}$ be an element of $G_{k}$. Then since $G_{k^{\prime}} \subseteq G_{k}$ is normal, it follows that $s\left(G_{k^{\prime}}\right) \subseteq D_{y} \cap\left(s(g) \cdot D_{y} \cdot s(g)^{-1}\right)$. Thus, by considering the image of $D_{y} \cap\left(s(g) \cdot D_{y} \cdot s(g)^{-1}\right) \subseteq \Pi_{k(Y) / k}^{\mathcal{C}}$ in $\Pi_{Y / k}^{\mathcal{C}}$, we conclude from Lemma 1.4 that $y$ is fixed by $g \in G_{k}$, hence [by allowing $g \in G_{k}$ to vary] that $y \in Y(k)$. This completes the proof of the implication $(2) \Rightarrow(1)$, hence also of Lemma 1.5.

Lemma 1.6. Let $X$ be a hyperbolic curve over $k$, s a pro-C Galois section of $X / k$ [cf. Definition 1.2], and $k^{\prime}(\subseteq \bar{k})$ a finite extension of $k$. Suppose that $k$ is either

(a) a finitely generated field of characteristic 0 or

(b) a $\boldsymbol{p}$-adic local field for some $p \in \Sigma(\mathcal{C})$.

Then the following conditions are equivalent:

(1) $s$ is geometric [cf. Definition 1.3].

(2) The pro-C Galois section $\left.s\right|_{G_{k^{\prime}}}$ of $X \otimes_{k} k^{\prime} / k^{\prime}$ determined by $s$ is geometric.

(3) For any open subgroup $H \subseteq \Pi_{X / k}^{\mathcal{C}}$ of $\Pi_{X / k}^{\mathcal{C}}$ containing the image of $s$, the [uniquely determined] smooth compactification of the finite étale covering of $X$ corresponding to $H \subseteq \Pi_{X / k}^{\mathcal{C}}$ admits a $k^{\prime}$-valued point.

Proof. This follows immediately from a similar argument to the argument applied in the proof of Lemma 1.5 [cf. also the proof of [7], Lemma 54 (respectively, [15], Proposition 2.8, (iv)), concerning the proof of the equivalence $(1) \Leftrightarrow(2)$ (respectively, $(1) \Leftrightarrow(3))$ ].

The following result was essentially proved in [12].

Proposition 1.7. Let $p$ be a prime number and $X$ a smooth curve over $k$. Suppose that $p \in \Sigma(\mathcal{C})$ [where we refer to the discussion entitled "Profinite groups" in $\S 0$ concerning the set $\Sigma(\mathcal{C})]$, and that $k$ is a $\boldsymbol{p}$-adic local field. Then any pro-C birational Galois section of $X / k[c f$. Definition 1.2] is geometric [cf. Definition 1.3].

Proof. It follows from the equivalence (1) $\Leftrightarrow(3)$ of Lemma 1.5 that, to verify Proposition 1.7, it suffices to verify that, for any open subgroup $H \subseteq \Pi_{k(X) / k}^{\mathcal{C}}$ of $\Pi_{k(X) / k}^{\mathcal{C}}$ containing the image of $s$, the [uniquely determined] smooth compactification of the normalization of $X$ in the finite extension of $k(X)$ corresponding to $H \subseteq \Pi_{k(X) / k}^{\mathcal{C}}$ admits a $k\left(\zeta_{p}\right)$-valued point, where we use the notation $\zeta_{p} \in \bar{k}$ to denote a 
primitive p-th root of unity. On the other hand, this follows immediately from [12], Theorem A, (2). This completes the proof of Proposition 1.7.

\section{LOCAL GEOMETRICITY OF BIRATIONAL GALOIS SECTIONS}

In the present $\S 2$, we discuss the notion of the local geometricity of birational Galois sections of smooth curves over number fields. In the present $\S 2$, let $\mathcal{C}$ be a full formation, $k$ a number field [where we refer to the discussion entitled "Numbers" in $\S 0$ concerning the term "number field"], $\bar{k}$ an algebraic closure of $k$, and $X$ a smooth curve over $k$ [where we refer to the discussion entitled "Curves" in $\S 0$ concerning the term "smooth curve"]. Write

$$
\mathfrak{o}_{k} \subseteq k
$$

for the ring of integers of $k$,

$$
\mathfrak{P}_{k}^{f}
$$

for the set of all nonarchimedean primes of $k$, and

$$
X^{\mathrm{cpt}}
$$

for the [uniquely determined] smooth compactification of $X$ over $k$. Moreover, for each $\mathfrak{p} \in \mathfrak{P}_{k}^{f}$, write

$$
k_{\mathfrak{p}}
$$

for the $\mathfrak{p}$-adic completion of $k$ and

$$
\mathfrak{o}_{\mathfrak{p}} \subseteq k_{\mathfrak{p}}
$$

for the ring of integers of $k_{\mathfrak{p}}$. For each $\mathfrak{p} \in \mathfrak{P}_{k}^{f}$, let us fix an algebraic closure $\bar{k}_{\mathfrak{p}}$ of $k_{\mathfrak{p}}$ containing $\bar{k}$ and write

$$
G_{\mathfrak{p}} \stackrel{\text { def }}{=} \operatorname{Gal}\left(\bar{k}_{\mathfrak{p}} / k_{\mathfrak{p}}\right) \subseteq G_{k} \stackrel{\text { def }}{=} \operatorname{Gal}(\bar{k} / k) \text {. }
$$

Definition 2.1. Let $s$ be a pro- $\mathcal{C}$ Galois section of $X / k$ [cf. Definition 1.2]. For a nonarchimedean prime $\mathfrak{p} \in \mathfrak{P}_{k}^{f}$ of $k$, we shall say that $s$ is geometric at $\mathfrak{p}$ if the pro-C Galois section of $X \otimes_{k} k_{\mathfrak{p}} / k_{\mathfrak{p}}$ naturally determined by $s$ [i.e., the pro-C Galois section of $X \otimes_{k} k_{\mathfrak{p}} / k_{\mathfrak{p}}$ determined by the natural isomorphism

$$
\Pi_{X \otimes_{k} k_{\mathfrak{p}} / k_{\mathfrak{p}}}^{\mathcal{C}} \stackrel{\sim}{\longrightarrow} \Pi_{X / k}^{\mathcal{C}} \times_{G_{k}} G_{\mathfrak{p}}
$$

and the composite

$$
\left.G_{\mathfrak{p}} \hookrightarrow G_{k} \stackrel{s}{\rightarrow} \Pi_{X / k}^{\mathcal{C}}\right]
$$

is geometric [cf. Definition 1.3]. For a subset $S \subseteq \mathfrak{P}_{k}^{f}$ of $\mathfrak{P}_{k}^{f}$, we shall say that $s$ is geometric at $S$ if, for each $\mathfrak{p} \in S, s$ is geometric at $\mathfrak{p}$. Finally, we shall say that $s$ is locally geometric if $s$ is geometric at $\mathfrak{P}_{k}^{f}$.

Remark 2.1.1. In the notation of Definition 2.1, it is immediate that if $s$ is geometric [cf. Definition 1.3], then $s$ is locally geometric. 
Definition 2.2. Let $S \subseteq \mathfrak{P}_{k}^{f}$ be a subset of $\mathfrak{P}_{k}^{f}$. Then we shall write

$$
\begin{gathered}
\left.\widetilde{\mathbb{A}}_{k}^{f}\right|_{S} \stackrel{\text { def }}{=} \prod_{\mathfrak{p} \in S} k_{\mathfrak{p}} ; \\
\left.\mathbb{A}_{k}^{f}\right|_{S} \stackrel{\text { def }}{=}\left\{\left(a_{\mathfrak{p}}\right)_{\mathfrak{p} \in S} \in\right. \\
\left.\left.\widetilde{\mathbb{A}}_{k}^{f}\right|_{S} \mid a_{\mathfrak{p}} \in \mathfrak{o}_{\mathfrak{p}} \text { for all but finitely many } \mathfrak{p} \in S\right\} ; \\
\left.\widetilde{\mathbb{A}}_{k}^{f} \stackrel{\text { def }}{=} \widetilde{\mathbb{A}}_{k}^{f}\right|_{\mathfrak{P}_{k}^{f}} ;\left.\mathbb{A}_{k}^{f} \stackrel{\text { def }}{=} \mathbb{A}_{k}^{f}\right|_{\mathfrak{P}_{k}^{f}} .
\end{gathered}
$$

Remark 2.2.1. Since $X^{\mathrm{cpt}}$ is proper over $k$, for any subset $S \subseteq \mathfrak{P}_{k}^{f}$ of $\mathfrak{P}_{k}^{f}$, the natural injection $X^{\mathrm{cpt}}\left(\left.\mathbb{A}_{k}^{f}\right|_{S}\right) \hookrightarrow X^{\mathrm{cpt}}\left(\left.\widetilde{\mathbb{A}}_{k}^{f}\right|_{S}\right)$ is bijective.

Definition 2.3. Let $s$ be a pro-C Galois section of $X / k$ [cf. Definition 1.2]. If $s$ is geometric at a nonarchimedean prime $\mathfrak{p} \in \mathfrak{P}_{k}^{f}$ of $k$ [cf. Definition 2.1], i.e., there exists a $k_{\mathfrak{p}}$-valued point $x_{\mathfrak{p}} \in X^{\mathrm{cpt}}\left(k_{\mathfrak{p}}\right)=$ $\left(X^{\mathrm{cpt}} \otimes_{k} k_{\mathfrak{p}}\right)\left(k_{\mathfrak{p}}\right)$ of $X^{\mathrm{cpt}}$ such that the image of the pro-C $\mathcal{C}$ Galois section of $X \otimes_{k} k_{\mathfrak{p}} / k_{\mathfrak{p}}$ naturally determined by $s$ is contained in a decomposition subgroup of $\Pi_{X \otimes_{k} k_{\mathfrak{p}} / k_{\mathfrak{p}}}^{\mathcal{C}}$ associated to $x_{\mathfrak{p}}$, then we shall refer to such a $k_{\mathfrak{p}}$-valued point " $x_{\mathfrak{p}}$ " of $X^{\mathrm{cpt}}$ as a $k_{\mathfrak{p}}$-valued point of $X^{\mathrm{cpt}}$ associated to $s$. If $s$ is geometric at a subset $S \subseteq \mathfrak{P}_{k}^{f}$ of $\mathfrak{P}_{k}^{f}$, then we shall refer to an $\left.\widetilde{\mathbb{A}}_{k}^{f}\right|_{S}$-valued point, or, equivalently [cf. Remark 2.2.1], an $\left.\mathbb{A}_{k}^{f}\right|_{S}$-valued point, of $X^{\mathrm{cpt}}$ determined by $k_{\mathfrak{p}}$-valued points of $X^{\mathrm{cpt}}$ associated to $s$ - where $\mathfrak{p}$ ranges over elements of $S$ - as an $\left.\widetilde{\mathbb{A}}_{k}^{f}\right|_{S^{-}}$ valued point, or, equivalently, an $\left.\mathbb{A}_{k}^{f}\right|_{S}$-valued point, of $X^{\mathrm{cpt}}$ associated to $s$.

Remark 2.3.1. In the notation of Definition 2.3, suppose that $s$ is geometric [cf. Definition 1.3], hence also locally geometric [cf. Definition 2.1; Remark 2.1.1]. Then it is immediate that there exists a $k$-rational point $x \in X^{\mathrm{cpt}}(k)$ of $X^{\mathrm{cpt}}$ such that, for each $\mathfrak{p} \in \mathfrak{P}_{k}^{f}$, the $k_{\mathfrak{p}}$-valued point of $X^{\mathrm{cpt}}$ determined by $x$ is a $k_{\mathfrak{p}}$-valued point of $X^{\mathrm{cpt}}$ associated to $s$. In particular, the $\mathbb{A}_{k}^{f}$-valued point of $X^{\mathrm{cpt}}$ determined by $x$ is an $\mathbb{A}_{k}^{f}$-valued point of $X^{\mathrm{cpt}}$ associated to $s$.

Note that if $\mathcal{C}$ contains all finite solvable groups, and $X$ is a hyperbolic curve over $k$ [where we refer to the discussion entitled "Curves" in $\S 0$ concerning the term "hyperbolic curve"], then it follows from Theorem 4.1 below that the converse holds, i.e., if $s$ is locally geometric, and there exists a $k$-rational point $x \in X^{\mathrm{cpt}}(k)$ of $X^{\mathrm{cpt}}$ such that, for each $\mathfrak{p} \in \mathfrak{P}_{k}^{f}$, the $k_{\mathfrak{p}}$-valued point of $X^{\mathrm{cpt}}$ determined by $x$ is a $k_{\mathfrak{p}}$-valued point of $X^{\mathrm{cpt}}$ associated to $s$, then $s$ is geometric.

Lemma 2.4. Let $s$ be a pro-C birational Galois section of $X / k[c f$. Definition 1.2] and $\mathfrak{p} \in \mathfrak{P}_{k}^{f}$. For an open subscheme $U \subseteq X^{\mathrm{cpt}}$ of $X^{\mathrm{cpt}}$, write

$$
s[U]
$$


for the pro-C Galois section of $U / k[c f$. Definition 1.2] naturally determined by $s$ [i.e., the pro-C Galois section of $U / k$ obtained as the composite of $s$ and the natural surjection $\left.\Pi_{k(X) / k}^{\mathcal{C}} \rightarrow \Pi_{U / k}^{\mathcal{C}}\right]$;

$$
s[U, \mathfrak{p}]
$$

for the pro-C Galois section of $U \otimes_{k} k_{\mathfrak{p}} / k_{\mathfrak{p}}$ naturally determined by $s$ [i.e., the pro-C Galois section of $U \otimes_{k} k_{\mathfrak{p}} / k_{\mathfrak{p}}$ determined by the natural isomorphism

$$
\Pi_{U \otimes_{k} k_{\mathfrak{p}} / k_{\mathfrak{p}}}^{\mathcal{C}} \stackrel{\sim}{\longrightarrow} \Pi_{U / k}^{\mathcal{C}} \times_{G_{k}} G_{\mathfrak{p}}
$$

and the composite

$$
\left.G_{\mathfrak{p}} \hookrightarrow G_{k} \stackrel{s}{\rightarrow} \Pi_{k(X) / k}^{\mathcal{C}} \rightarrow \Pi_{U / k}^{\mathcal{C}}\right] .
$$

Then the following conditions are equivalent:

(1) There exists a $k_{\mathfrak{p}}$-valued point $x_{\mathfrak{p}} \in X^{\mathrm{cpt}}\left(k_{\mathfrak{p}}\right)=\left(X^{\mathrm{cpt}} \otimes_{k} k_{\mathfrak{p}}\right)\left(k_{\mathfrak{p}}\right)$ of $X^{\mathrm{cpt}}$ such that, for any open subscheme $U \subseteq X^{\mathrm{cpt}}$ of $X^{\mathrm{cpt}}$, the image of the pro-C Galois section $s[U, \mathfrak{p}]$ of $U \otimes_{k} k_{\mathfrak{p}} / k_{\mathfrak{p}}$ is contained in a decomposition subgroup of $\Pi_{U \otimes_{k} k_{\mathfrak{p}} / k_{\mathfrak{p}}}^{\mathcal{C}}$ associated to $x_{\mathfrak{p}}$.

(2) For any open subscheme $U \subseteq X^{\mathrm{cpt}}$ of $X^{\mathrm{cpt}}$, the pro-C Galois section $s[U]$ of $U / k$ is geometric at $\mathfrak{p}[c f$. Definition 2.1], i.e., the pro-C Galois section $s[U, \mathfrak{p}]$ of $U \otimes_{k} k_{\mathfrak{p}} / k_{\mathfrak{p}}$ is geometric [or, equivalently, the image of $s[U, \mathfrak{p}]$ is contained in a decomposition subgroup of $\Pi_{U \otimes_{k} k_{\mathfrak{p}} / k_{\mathfrak{p}}}^{\mathcal{C}}$ associated to a $k_{\mathfrak{p}}$-rational point of $\left.X^{\mathrm{cpt}} \otimes_{k} k_{\mathfrak{p}}\right]$.

(3) For any open subgroup $H \subseteq \Pi_{k(X) / k}^{\mathcal{C}}$ of $\Pi_{k(X) / k}^{\mathcal{C}}$ containing the image of $s$, the [uniquely determined] smooth compactification of the normalization of $X$ in the finite extension of $k(X)$ corresponding to $H \subseteq \Pi_{k(X) / k}^{\mathcal{C}}$ admits a $k_{\mathfrak{p}}$-valued point.

(4) The image of the homomorphism $G_{\mathfrak{p}} \rightarrow \Pi_{k(X) / k}^{\mathcal{C}} \times{ }_{G_{k}} G_{\mathfrak{p}}$ induced by $s$ is contained in the image of a decomposition subgroup of $\Pi_{k_{\mathfrak{p}}\left(X \otimes_{k} k_{\mathfrak{p}}\right) / k_{\mathrm{p}}}^{\mathcal{C}}$ associated to a [necessarily $k_{\mathfrak{p}}$-rational] closed point of $X^{\mathrm{cpt}} \otimes_{k} k_{\mathfrak{p}}$ by the natural surjection $\Pi_{k_{\mathfrak{p}}\left(X \otimes_{k} k_{\mathfrak{p}}\right) / k_{\mathfrak{p}}}^{\mathcal{C}} \rightarrow \Pi_{k(X) / k}^{\mathcal{C}} \times{ }_{G_{k}} G_{\mathfrak{p}}$.

Proof. The implications $(4) \Rightarrow(1) \Rightarrow(2)$ are immediate. Next, we verify the implication $(2) \Rightarrow(3)$. Suppose that condition (2) is satisfied. Write $Y$ for the [uniquely determined] smooth compactification of the normalization of $X$ in the finite extension of $k(X)$ corresponding to $H \subseteq \Pi_{k(X) / k}^{\mathcal{C}}$; thus, $\Pi_{k(Y) / k}^{\mathcal{C}}=H \subseteq \Pi_{k(X) / k}^{\mathcal{C}}$. Then it is immediate that there exists an open subscheme $U \subseteq X^{\mathrm{cpt}}$ of $X^{\mathrm{cpt}}$ such that the finite morphism $Y \rightarrow X^{\mathrm{cpt}}$ determines a connected finite étale covering $V \stackrel{\text { def }}{=} Y \times_{X^{\mathrm{cpt}}} U \rightarrow U$ which corresponds to an open subgroup $\Pi_{V / k}^{\mathcal{C}} \subseteq \Pi_{U / k}^{\mathcal{C}}$ of $\Pi_{U / k}^{\mathcal{C}}$. On the other hand, since $H=\Pi_{k(Y) / k}^{\mathcal{C}} \subseteq \Pi_{k(X) / k}^{\mathcal{C}}$ contains the image of $s$, one verifies easily that the open subgroup $\Pi_{V / k}^{\mathcal{C}} \subseteq \Pi_{U / k}^{\mathcal{C}}$ contains the image of $s[U]$. Thus, it follows immediately from condition (2) that $Y\left(k_{\mathfrak{p}}\right) \neq \emptyset$. This completes the proof of the implication $(2) \Rightarrow(3)$. 
Finally, we verify the implication $(3) \Rightarrow(4)$. Suppose that condition (3) is satisfied. Write $\left\{H_{i}\right\}_{i \in I}$ for the projective system of open subgroups $H_{i} \subseteq \Pi_{k(X) / k}^{\mathcal{C}}$ of $\Pi_{k(X) / k}^{\mathcal{C}}$ containing the image of $s$. [Thus, we have that $\bigcap_{i} H_{i}=\operatorname{Im}(s)$.] For each $i \in I$, write $X_{i}$ for the [uniquely determined] smooth compactification of the normalization of $X$ in the finite extension of $k(X)$ corresponding to $H_{i}$; thus, $\Pi_{k\left(X_{i}\right) / k}^{\mathcal{C}}=H_{i} \subseteq \Pi_{k(X) / k}^{\mathcal{C}}$, and we have a projective system $\left\{X_{i}\right\}_{i \in I}$ of projective smooth curves over $k$ [with finite morphisms over $k$ ], hence also a projective system $\left\{X_{i}\left(k_{\mathfrak{p}}\right)\right\}_{i \in I}$ of compact sets with continuous maps [cf. the proof of the implication $(3) \Rightarrow(2)$ of Lemma 1.5]. Thus, it follows immediately from condition (3) that $\lim _{i} X_{i}\left(k_{\mathfrak{p}}\right) \neq \emptyset$. Let $x_{\infty} \in \lim X_{i}\left(k_{\mathfrak{p}}\right)$ be an element of $\lim _{i} X_{i}\left(k_{\mathfrak{p}}\right)$. Then one verifies easily that $x_{\infty}$ determines, for each $\overleftarrow{i} \in I$, a $k_{\mathfrak{p}}$-rational point $x_{i}$ of $X_{i} \otimes_{k} k_{\mathfrak{p}}$ and a decomposition subgroup $D_{i} \subseteq \Pi_{k_{\mathfrak{p}}\left(X_{i} \otimes_{k} k_{\mathfrak{p}}\right) / k_{\mathfrak{p}}}^{\mathcal{C}}$ of $\Pi_{k_{\mathfrak{p}}\left(X_{i} \otimes_{k} k_{\mathfrak{p}}\right) / k_{\mathfrak{p}}}^{\mathcal{C}}$ associated to $x_{i}$ such that if we write $D_{i}^{H} \subseteq \Pi_{k\left(X_{i}\right) / k}^{\mathcal{C}} \times{ }_{G_{k}}$ $G_{\mathfrak{p}}=H_{i} \times_{G_{k}} G_{\mathfrak{p}}$ for the image of $D_{i} \subseteq \Pi_{k_{\mathfrak{p}}\left(X_{i} \otimes_{k} k_{\mathfrak{p}}\right) / k_{\mathfrak{p}}}^{\mathcal{C}}$ by the natural surjection $\Pi_{k_{\mathfrak{p}}\left(X_{i} \otimes_{k} k_{\mathfrak{p}}\right) / k_{\mathfrak{p}}}^{\mathcal{C}} \rightarrow \Pi_{k\left(X_{i}\right) / k}^{\Sigma} \times_{G_{k}} G_{\mathfrak{p}}=H_{i} \times_{G_{k}} G_{\mathfrak{p}}$, then the $D_{i}^{H \prime}$ s are compatible with the various inclusions appearing in the projective system $\left\{H_{i}\right\}_{i \in I}$, and, moreover, the image of the composite

$$
\bigcap_{i} D_{i}^{H} \hookrightarrow \Pi_{k(X) / k}^{\mathcal{C}} \rightarrow G_{k}
$$

coincides with $G_{\mathfrak{p}} \subseteq G_{k}$. On the other hand, since $\bigcap_{i} D_{i}^{H} \subseteq \bigcap_{i} H_{i}=$ $\operatorname{Im}(s)$, one verifies easily that $\operatorname{Im}\left(\left.s\right|_{G_{\mathfrak{p}}}\right) \subseteq \bigcap_{i} D_{i}^{H} ;$ in particular, $\operatorname{Im}\left(\left.s\right|_{G_{\mathfrak{p}}}\right)$ is contained in " $D_{i}^{H \text { " }}$ in the case where we take " $H_{i}$ " to be $\Pi_{k(X) / k}^{\mathcal{C}}$ " i.e., condition (4) is satisfied. This completes the proof of the implication $(3) \Rightarrow(4)$, hence also of Lemma 2.4.

Definition 2.5. Let $s$ be a pro-C $\mathcal{C}$ birational Galois section of $X / k$ [cf. Definition 1.2]. For a nonarchimedean prime $\mathfrak{p} \in \mathfrak{P}_{k}^{f}$ of $k$, we shall say that $s$ is geometric at $\mathfrak{p}$ if the pair $(s, \mathfrak{p})$ satisfies equivalent conditions (1), (2), (3), and (4) of Lemma 2.4. For a subset $S \subseteq \mathfrak{P}_{k}^{f}$ of $\mathfrak{P}_{k}^{f}$, we shall say that $s$ is geometric at $S$ if, for each $\mathfrak{p} \in S, s$ is geometric at $\mathfrak{p}$. Finally, we shall say that $s$ is locally geometric if $s$ is geometric at $\mathfrak{P}_{k}^{f}$.

Remark 2.5.1. In the notation of Definition 2.5, it is immediate that if $s$ is geometric [cf. Definition 1.3], then $s$ is locally geometric.

Definition 2.6. Let $s$ be a pro-C birational Galois section of $X / k$ [cf. Definition 1.2]. If $s$ is geometric at a nonarchimedean prime $\mathfrak{p} \in$ $\mathfrak{P}_{k}^{f}$ of $k$ [cf. Definition 2.5], i.e., the pair $(s, \mathfrak{p})$ satisfies condition (1) of Lemma 2.4 , then we shall refer to a $k_{\mathfrak{p}}$-valued point " $x_{\mathfrak{p}}$ " of $X^{\mathrm{cpt}}$ appearing in condition (1) of Lemma 2.4 as a $k_{\mathfrak{p}}$-valued point of $X^{\mathrm{cpt}}$ associated to $s$. If $s$ is geometric at a subset $S \subseteq \mathfrak{P}_{k}^{f}$ of $\mathfrak{P}_{k}^{f}$, then we shall 
refer to an $\left.\widetilde{\mathbb{A}}_{k}^{f}\right|_{S}$-valued point, or, equivalently [cf. Remark 2.2.1], an $\left.\mathbb{A}_{k}^{f}\right|_{S}$-valued point, of $X^{\mathrm{cpt}}$ determined by $k_{\mathrm{p}}$-valued points of $X^{\mathrm{cpt}}$ associated to $s$ - where $\mathfrak{p}$ ranges over elements of $S-$ as an $\left.\widetilde{\mathbb{A}}_{k}^{f}\right|_{S^{-}}$ valued point, or, equivalently, an $\left.\mathbb{A}_{k}^{f}\right|_{S}$-valued point, of $X^{\mathrm{cpt}}$ associated to $s$.

Remark 2.6.1. In the notation of Definition 2.6, suppose that $s$ is geometric [cf. Definition 1.3], hence also locally geometric [cf. Definition 2.5; Remark 2.5.1]. Then it is immediate that there exists a $k$-rational point $x \in X^{\mathrm{cpt}}(k)$ of $X^{\mathrm{cpt}}$ such that, for each $\mathfrak{p} \in \mathfrak{P}_{k}^{f}$, the $k_{\mathrm{p}}$-valued point of $X^{\mathrm{cpt}}$ determined by $x$ is a $k_{\mathrm{p}}$-valued point of $X^{\mathrm{cpt}}$ associated to $s$. In particular, the $\mathbb{A}_{k}^{f}$-valued point of $X^{\mathrm{cpt}}$ determined by $x$ is an $\mathbb{A}_{k}^{f}$-valued point of $X^{\mathrm{cpt}}$ associated to $s$.

Note that if $\mathcal{C}$ contains all finite solvable groups, then it follows from Theorem 4.1 below that the converse holds, i.e., if $s$ is locally geometric, and there exists a $k$-rational point $x \in X^{\mathrm{cpt}}(k)$ of $X^{\mathrm{cpt}}$ such that, for each $\mathfrak{p} \in \mathfrak{P}_{k}^{f}$, the $k_{\mathfrak{p}}$-valued point of $X^{\mathrm{cpt}}$ determined by $x$ is a $k_{\mathfrak{p}}$-valued point of $X^{\mathrm{cpt}}$ associated to $s$, then $s$ is geometric.

Lemma 2.7. Let s be a pro-C birational Galois section (respectively, pro-C Galois section) of X/k [cf. Definition 1.2] and $S \subseteq \mathfrak{P}_{k}^{f}$ a subset of $\mathfrak{P}_{k}^{f}$. Suppose that $s$ is geometric at $\boldsymbol{S}[c f$. Definition 2.5 (respectively, Definition 2.1)], and that, for each $\mathfrak{p} \in S$, the residue characteristic of $\mathfrak{p}$ is $\in \Sigma(\mathcal{C})$ [where we refer to the discussion entitled "Profinite groups" in $\S 0$ concerning the set $\Sigma(\mathcal{C})]$. Suppose, moreover, that if $s$ is a pro-C Galois section of $X / k$, then $X$ is a hyperbolic curve over $k$ [where we refer to the discussion entitled "Curves" in $§ 0$ concerning the term "hyperbolic curve"]. Then an $\left.\mathbb{A}_{k}^{f}\right|_{S}$-valued point of $X^{\mathrm{cpt}}$ associated to $s[c f$. Definition 2.6 (respectively, Definition 2.3)] is uniquely determined by $s$.

Proof. Observe that, to verify Lemma 2.7, by replacing $S$ by a subset of $S$ of cardinality 1, we may assume without loss of generality that $S=\{\mathfrak{p}\}$ for some $\mathfrak{p} \in \mathfrak{P}_{k}^{f}$. Then the uniqueness in question follows immediately from Lemma 1.4. This completes the proof of Lemma 2.7 .

Lemma 2.8. Let $s$ be a pro-C birational Galois section of $X / k[c f$. Definition 1.2], $S \subseteq \mathfrak{P}_{k}^{f}$ a subset of $\mathfrak{P}_{k}^{f}$, and $x_{\mathbb{A}} \in X^{\mathrm{cpt}}\left(\left.\mathbb{A}_{k}^{f}\right|_{S}\right)$ an $\left.\mathbb{A}_{k}^{f}\right|_{S}$-valued point of $X^{\mathrm{cpt}}$. Suppose that $s$ is geometric at $\boldsymbol{S}$ [cf. Definition 2.5]. Write $s[X]$ for the pro-C Galois section of $X / k[c f$. Definition 1.2] naturally determined by $s$. Then the following hold:

(i) $s[X]$ is geometric at $\boldsymbol{S}$ [cf. Definition 2.1].

(ii) If $x_{\mathbb{A}} \in X^{\mathrm{cpt}}\left(\left.\mathbb{A}_{k}^{f}\right|_{S}\right)$ is an $\left.\mathbb{A}_{k}^{f}\right|_{S}$-valued point of $X^{\mathrm{cpt}}$ associated to $s$ [cf. Definition 2.6], then $x_{\mathbb{A}} \in X^{\mathrm{cpt}}\left(\left.\mathbb{A}_{k}^{f}\right|_{S}\right)$ is an $\left.\mathbb{A}_{k}^{f}\right|_{S^{-}}$valued point of $X^{\mathrm{cpt}}$ associated to $s[X]$ [cf. Definition 2.3; assertion (i)]. 
(iii) Suppose, moreover, that, for each $\mathfrak{p} \in S$, the residue characteristic of $\mathfrak{p}$ is $\in \Sigma(\mathcal{C})$ [where we refer to the discussion entitled "Profinite groups" in $\S 0$ concerning the set $\Sigma(\mathcal{C})]$, and that $X$ is a hyperbolic curve over $k$. Then it holds that $x_{\mathbb{A}} \in X^{\mathrm{cpt}}\left(\left.\mathbb{A}_{k}^{f}\right|_{S}\right)$ is an $\left.\mathbb{A}_{k}^{f}\right|_{S^{-}}$ valued point of $X^{\mathrm{cpt}}$ associated to $s$ if and only if $x_{\mathbb{A}} \in X^{\mathrm{cpt}}\left(\left.\mathbb{A}_{k}^{f}\right|_{S}\right)$ is an $\left.\mathbb{A}_{k}^{f}\right|_{s}$-valued point of $X^{\mathrm{cpt}}$ associated to $s[X][c f$. assertion (i)].

Proof. Assertions (i), (ii) follow immediately from the various definitions involved. Assertion (iii) follows immediately from Lemma 2.7, together with assertion (ii). This completes the proof of Lemma 2.8.

The following result was essentially proved in [12].

Proposition 2.9. Let $s$ be a pro-C birational Galois section of $X / k[c f$. Definition 1.2] and $S \subseteq \mathfrak{P}_{k}^{f}$ a subset of $\mathfrak{P}_{k}^{f}$ such that, for each $\mathfrak{p} \in S$, the residue characteristic of $\mathfrak{p}$ is $\in \Sigma(\mathcal{C})$ [where we refer to the discussion entitled "Profinite groups" in $\S 0$ concerning the set $\Sigma(\mathcal{C})]$. Then $s$ is geometric at $\boldsymbol{S}$ [cf. Definition 2.5]. In particular, s determines a unique $\left.\mathbb{A}_{k}^{f}\right|_{S}$-valued point of $X^{\mathrm{cpt}}[c f$. Definition 2.6].

Proof. If $s$ is geometric at $S$, then the uniqueness of an $\left.\mathbb{A}_{k}^{f}\right|_{S}$-valued point of $X^{\mathrm{cpt}}$ associated to $s$ follows from Lemma 2.7. Thus, to verify Proposition 2.9, it suffices to verify that $s$ is geometric at $S$. Moreover, it follows immediately from the various definitions involved that, to verify that $s$ is geometric at $S$, by replacing $S$ by a subset of $S$ of cardinality 1, we may assume without loss of generality that $S=\{\mathfrak{p}\}$ for some $\mathfrak{p} \in \mathfrak{P}_{k}^{f}$, whose residue characteristic we denote by $p$. Thus, it follows from Lemma 2.10 below [cf. condition (5) of Lemma 2.10 below] that, to verify Proposition 2.9, it suffices to verify that, for any open subgroup $H \subseteq \Pi_{k(X) / k}^{\mathcal{C}}$ of $\Pi_{k(X) / k}^{\mathcal{C}}$ containing the image of $s$, the [uniquely determined] smooth compactification $Y$ of the normalization of $X$ in the finite extension of $k(X)$ corresponding to $H \subseteq \Pi_{k(X) / k}^{\mathcal{C}}$ admits a $k_{\mathfrak{p}}\left(\zeta_{p}\right)$-valued point, where we use the notation $\zeta_{p} \in \bar{k}$ to denote a primitive $p$-th root of unity. On the other hand, by considering the restriction of the pro- $\mathcal{C}$ birational Galois section of $Y / k$ naturally determined by $s$ to the closed subgroup $\operatorname{Gal}\left(\bar{k} / k\left(\zeta_{p}\right)^{\mathrm{h}}\right)$ of $G_{k}$, where we write $k\left(\zeta_{p}\right)^{\mathrm{h}} \subseteq \bar{k}$ for the algebraic closure of $k\left(\zeta_{p}\right)$ in $k_{\mathfrak{p}}\left(\zeta_{p}\right)$, we conclude from [12], Theorem B, (2), that $Y\left(k\left(\zeta_{p}\right)^{\mathrm{h}}\right) \neq \emptyset$, hence also that $Y\left(k_{\mathfrak{p}}\left(\zeta_{p}\right)\right) \neq \emptyset$. This completes the proof of Proposition 2.9 .

Lemma 2.10. In the notation of Lemma 2.4, suppose, moreover, that the residue characteristic of $\mathfrak{p}$ is $\in \Sigma(\mathcal{C})$ [where we refer to the discussion entitled "Profinite groups" in $\S 0$ concerning the set $\Sigma(\mathcal{C})$ ]. Let $k_{\mathfrak{p}}^{\prime}\left(\subseteq \bar{k}_{\mathfrak{p}}\right)$ be a finite extension of $k_{\mathfrak{p}}$. Then equivalent conditions (1), (2), (3), and (4) of Lemma 2.4 are equivalent to the following conditions: 
(5) For any open subgroup $H \subseteq \Pi_{k(X) / k}^{\mathcal{C}}$ of $\Pi_{k(X) / k}^{\mathcal{C}}$ containing the image of $s$, the [uniquely determined] smooth compactification of the normalization of $X$ in the finite extension of $k(X)$ corresponding to $H \subseteq \Pi_{k(X) / k}^{\mathcal{C}}$ admits a $k_{\mathfrak{p}}^{\prime}$-valued point.

(6) The image of the composite of the natural inclusion $\mathrm{Gal}\left(\bar{k}_{\mathfrak{p}} / k_{\mathfrak{p}}^{\prime}\right) \hookrightarrow$ $G_{\mathfrak{p}}$ and the homomorphism $G_{\mathfrak{p}} \rightarrow \Pi_{k(X) / k}^{\mathcal{C}} \times_{G_{k}} G_{\mathfrak{p}}$ induced by $s$ is contained in the image of a decomposition subgroup of $\Pi_{k_{\mathfrak{p}}\left(X \otimes_{k} k_{\mathfrak{p}}\right) / k_{\mathfrak{p}}}^{\mathcal{C}}$ associated to a closed point of $X^{\mathrm{cpt}} \otimes_{k} k_{\mathfrak{p}}$ [necessarily defined over $a$ subfield of $k_{\mathfrak{p}}^{\prime}$ by the natural surjection $\prod_{k_{\mathfrak{p}}\left(X \otimes_{k} k_{\mathfrak{p}}\right) / k_{\mathfrak{p}}}^{\mathcal{C}} \rightarrow \Pi_{k(X) / k}^{\mathcal{C}} \times_{G_{k}}$ $G_{\mathfrak{p}}$.

Proof. The implication $(3) \Rightarrow(5)$ is immediate. Moreover, by applying the implication $(3) \Rightarrow(4)$ of Lemma 2.4 to the restriction of $s$ to a suitable open subgroup of $G_{k}$, we conclude that the implication $(5) \Rightarrow(6)$ holds. Finally, we verify the implication $(6) \Rightarrow(3)$. Suppose that condition (6) is satisfied. Then, to verify the implication $(6) \Rightarrow(3)$, by replacing $k_{\mathfrak{p}}^{\prime}$ by a finite extension of $k_{\mathfrak{p}}^{\prime}$, we may assume without loss of generality that $k_{\mathfrak{p}}^{\prime}$ is Galois over $k_{\mathfrak{p}}$. Moreover, to verify the implication $(6) \Rightarrow(3)$, by replacing $\Pi_{k(X) / k}^{\mathcal{C}}$ by an open subgroup of $\Pi_{k(X) / k}^{\mathcal{C}}$ containing the image of $s$, we may assume without loss of generality that $X$ is of genus $\geq 2$. Write $Y$ for the [uniquely determined] smooth compactification of the normalization of $X$ in the finite extension of $k(X)$ corresponding to $H \subseteq \Pi_{k(X) / k}^{\mathcal{C}}$; thus, $\Pi_{k(Y) / k}^{\mathcal{C}}=H \subseteq \Pi_{k(X) / k}^{\mathcal{C}}$. Now it follows immediately from condition (6) that there exist a closed point $y$ of $Y \otimes_{k} k_{\mathfrak{p}}$ which is defined over a subfield of $k_{\mathfrak{p}}^{\prime}$ and a decomposition subgroup $D_{y} \subseteq \Pi_{k_{\mathfrak{p}}\left(Y \otimes_{k} k_{\mathfrak{p}}\right) / k_{\mathfrak{p}}}^{\mathcal{C}}$ of $\Pi_{k_{\mathfrak{p}}\left(Y \otimes_{k} k_{\mathfrak{p}}\right) / k_{\mathfrak{p}}}^{\mathcal{C}}$ associated to $y$ such that if we write $D_{y}^{H} \subseteq \Pi_{k(Y) / k}^{\mathcal{C}} \times_{G_{k}} G_{\mathfrak{p}}=H \times_{G_{k}} G_{\mathfrak{p}}$ for the image of $D_{y} \subseteq$ $\Pi_{k_{\mathfrak{p}}\left(Y \otimes_{k} k_{\mathfrak{p}}\right) / k_{\mathfrak{p}}}^{\mathcal{C}}$ by the natural surjection $\Pi_{k_{\mathfrak{p}}\left(Y \otimes_{k} k_{\mathfrak{p}}\right) / k_{\mathfrak{p}}}^{\mathcal{C}} \rightarrow \Pi_{k(Y) / k}^{\mathcal{C}} \times_{G_{k}}$ $G_{\mathfrak{p}}=H \times_{G_{k}} G_{\mathfrak{p}}$, then $s\left(\operatorname{Gal}\left(\bar{k}_{\mathfrak{p}} / k_{\mathfrak{p}}^{\prime}\right)\right) \subseteq D_{y}^{H}$. Let $g \in G_{\mathfrak{p}}$ be an element of $G_{\mathfrak{p}}$. Then since $\operatorname{Gal}\left(\bar{k}_{\mathfrak{p}} / k_{\mathfrak{p}}^{\prime}\right) \subseteq G_{\mathfrak{p}}$ is normal, it follows that $s\left(\operatorname{Gal}\left(\bar{k}_{\mathfrak{p}} / k_{\mathfrak{p}}^{\prime}\right)\right) \subseteq D_{y}^{H} \cap\left(s(g) \cdot D_{y}^{H} \cdot s(g)^{-1}\right)$. Thus, by considering the image of $D_{y}^{H} \cap\left(s(g) \cdot D_{y}^{H} \cdot s(g)^{-1}\right) \subseteq \Pi_{k(Y) / k}^{\mathcal{C}} \times_{G_{k}} G_{\mathfrak{p}}=H \times_{G_{k}} G_{\mathfrak{p}}$ in $\Pi_{Y / k}^{\mathcal{C}} \times_{G_{k}} G_{\mathfrak{p}} \stackrel{\sim}{\leftarrow} \Pi_{Y \otimes_{k} k_{\mathfrak{p}} / k_{\mathfrak{p}}}^{\mathcal{C}}$, we conclude from Lemma 1.4 that $y$ is fixed by $g \in G_{\mathfrak{p}}$, hence [by allowing $g \in G_{\mathfrak{p}}$ to vary] that $y \in Y\left(k_{\mathfrak{p}}\right)$. This completes the proof of the implication $(6) \Rightarrow(3)$, hence also of Lemma 2.10.

Proposition 2.11. Suppose that $X$ is a hyperbolic curve over $k$. Let $s$ be a pro-C Galois section of $X / k$ [cf. Definition 1.2] and $S \subseteq \mathfrak{P}_{k}^{f}$ a subset of $\mathfrak{P}_{k}^{f}$ such that, for each $\mathfrak{p} \in S$, the residue characteristic of $\mathfrak{p}$ is $\in \Sigma(\mathcal{C})$ [where we refer to the discussion entitled "Profinite groups" in $\S 0$ concerning the set $\Sigma(\mathcal{C})]$. Suppose that $s$ arises from a pro-C birational 
Galois section of $X / k$ [cf. Definition 1.2]. Then $s$ is geometric at $S$ [cf. Definition 2.1]. In particular, s determines a unique $\left.\mathbb{A}_{k}^{f}\right|_{S}$-valued point of $X^{\mathrm{cpt}}[c f$. Definition 2.3].

Proof. The fact that $s$ is geometric at $S$ follows immediately from Proposition 2.9, together with Lemma 2.8, (i). The fact that $s$ determines a unique $\left.\mathbb{A}_{k}^{f}\right|_{S}$-valued point of $X^{\mathrm{cpt}}$ follows immediately from Lemma 2.7 . This completes the proof of Proposition 2.11.

\section{GALOIS SECTIONS OF TORI THAT LOCALLY ARISE FROM POINTS}

In the present $\S 3$, we discuss Galois sections of tori that locally arise from points. We maintain the notation of the preceding $\S 2$. Let $\Sigma \subseteq$ $\mathfrak{P r i m e s}$ be a nonempty subset of $\mathfrak{P r i m e s}$ [where we refer to the discussion entitled "Numbers" in $\S 0$ concerning the set $\mathfrak{P r i m e s}]$. Write

$$
\begin{gathered}
\operatorname{Div}\left(\mathfrak{o}_{k}\right) \stackrel{\text { def }}{=} \bigoplus_{\mathfrak{p} \in \mathfrak{P}_{k}^{f}} \mathbb{Z} ; \\
\operatorname{Pic}\left(\mathfrak{o}_{k}\right) \stackrel{\text { def }}{=} \operatorname{Pic}\left(\operatorname{Spec} \mathfrak{o}_{k}\right) ; \\
d_{k}
\end{gathered}
$$

for the minimal positive integer such that $d_{k} \cdot \operatorname{Pic}\left(\mathfrak{o}_{k}\right)=\{0\}$;

$$
\mathbb{G}_{m, \mathbb{Z}} \stackrel{\text { def }}{=} \mathbb{P}_{\mathbb{Z}}^{1} \backslash\{0, \infty\}=\operatorname{Spec} \mathbb{Z}\left[u^{ \pm 1}\right] ;
$$

for each $\mathfrak{p} \in \mathfrak{P}_{k}^{f}$,

$$
v_{\mathfrak{p}}: k^{\times} \longrightarrow \mathbb{Z}
$$

for the $\mathfrak{p}$-adic valuation which induces a surjection $k_{\mathfrak{p}}^{\times} \rightarrow \mathbb{Z}$;

$$
\begin{aligned}
\operatorname{div}_{k}: \quad k^{\times} & \longrightarrow \sum_{\operatorname{Div}\left(\mathfrak{o}_{k}\right)} \\
a & \mapsto \sum_{\mathfrak{p} \in \mathfrak{P}_{k}^{f}} v_{\mathfrak{p}}(a) \cdot \mathfrak{p} .
\end{aligned}
$$

[Thus, we have an exact sequence of modules

$$
\left.0 \longrightarrow \mathfrak{o}_{k}^{\times} \longrightarrow k^{\times} \stackrel{\operatorname{div}_{k}}{\longrightarrow} \operatorname{Div}\left(\mathfrak{o}_{k}\right) \longrightarrow \operatorname{Pic}\left(\mathfrak{o}_{k}\right) \longrightarrow 0 .\right]
$$

Write, moreover, for a ring $R$,

$$
\mathbb{G}_{m, R} \stackrel{\text { def }}{=} \mathbb{G}_{m, \mathbb{Z}} \otimes_{\mathbb{Z}} R .
$$

Let us identify $\mathbb{G}_{m, R}(R)$ with $R^{\times}$by the invertible function $u \in R\left[u^{ \pm 1}\right]^{\times}$:

$$
\mathbb{G}_{m, R}(R) \simeq R^{\times} .
$$

Definition 3.1. Let $M$ be a module. Then we shall write

$$
M[\Sigma] \stackrel{\text { def }}{=} \underset{\longleftarrow}{\longleftarrow} M / n M
$$

- where $n$ ranges over positive $\Sigma$-integers [where we refer to the discussion entitled "Numbers" in $\S 0$ concerning the term " $\Sigma$-integer"]. Thus, if, moreover, for any positive $\Sigma$-integer $n$, the module $M / n M$ is finite [e.g., $M$ is finitely generated], then $M[\Sigma]$ is the pro- $\Sigma$ completion of $M$. 


\section{Lemma 3.2.}

(i) For every [not necessarily algebraic] extension $k^{\prime}$ of $k$, if we write

$$
\mathrm{GS}^{\Sigma}\left(\mathbb{G}_{m, k^{\prime}} / k^{\prime}\right)
$$

for the set of conjugacy classes of pro- $\Sigma$ Galois sections of $\mathbb{G}_{m, k^{\prime}} / k^{\prime}$ [cf. Definition 1.2], then the map

$$
\mathrm{GS}^{\Sigma}\left(\mathbb{G}_{m, k^{\prime}} / k^{\prime}\right) \longrightarrow H^{1}\left(k^{\prime}, \Delta_{\mathbb{G}_{m, k} / k}^{\Sigma}\right)
$$

determined by the natural isomorphism

$$
\Delta_{\mathbb{G}_{m, k^{\prime}} / k^{\prime}}^{\Sigma} \stackrel{\sim}{\longrightarrow} \Delta_{\mathbb{G}_{m, k} / k}^{\Sigma}
$$

induced by $k \hookrightarrow k^{\prime}$ and the map

$$
\mathrm{GS}^{\Sigma}\left(\mathbb{G}_{m, k^{\prime}} / k^{\prime}\right) \longrightarrow H^{1}\left(k^{\prime}, \Delta_{\mathbb{G}_{m, k^{\prime}}}^{\Sigma} / k^{\prime}\right)
$$

given by mapping an element $s \in \mathrm{GS}^{\Sigma}\left(\mathbb{G}_{m, k^{\prime}} / k^{\prime}\right)$ to the element of $H^{1}\left(k^{\prime}, \Delta_{\mathbb{G}_{m, k^{\prime}} / k^{\prime}}^{\Sigma}\right)$ obtained by considering the difference of $s$ and the element $\mathrm{GS}^{\Sigma}\left(\mathbb{G}_{m, k^{\prime}} / k^{\prime}\right)$ arising from the $k$-rational point $1 \in$ $\left(k^{\prime}\right)^{\times} \simeq \mathbb{G}_{m, k^{\prime}}\left(k^{\prime}\right)$ is bijective.

(ii) There exists a natural isomorphism $\Delta_{\mathbb{G}_{m, k} / k}^{\Sigma} \stackrel{\sim}{\rightarrow} \widehat{\mathbb{Z}}^{\Sigma}(1)$ [where " $(1)$ " denotes a Tate twist] such that, for every [not necessarily algebraic] extension $k^{\prime}$ of $k$, the following diagram of sets commutes:

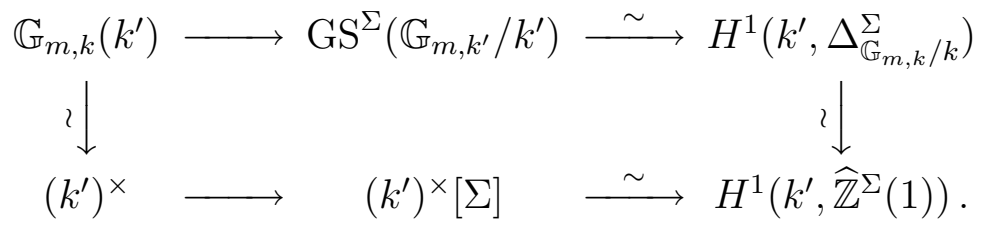

Here, the left-hand upper horizontal arrow is the natural map given by mapping a $k^{\prime}$-rational point of $\mathbb{G}_{m, k^{\prime}}$ to the conjugacy class of a pro- $\Sigma$ Galois section of $\mathbb{G}_{m, k^{\prime}} / k^{\prime}$ associated to the $k^{\prime}$-rational point, the right-hand upper horizontal arrow is the bijection of (i), the left-hand vertical arrow is the natural identification by the fixed invertible function $u$, the right-hand vertical arrow is the isomorphism induced by the isomorphism in question $\Delta_{\mathbb{G}_{m, k} / k}^{\Sigma} \stackrel{\sim}{\rightarrow} \widehat{\mathbb{Z}}^{\Sigma}(1)$, the left-hand lower horizontal arrow is the natural homomorphism [cf. Definition 3.1], and the right-hand lower horizontal arrow is the natural isomorphism given by the Kummer theory.

(iii) Let $S \subseteq \mathfrak{P}_{k}^{f}$ be a subset of $\mathfrak{P}_{k}^{f}$. Then there exists a natural isomorphism between the commutative diagram of sets

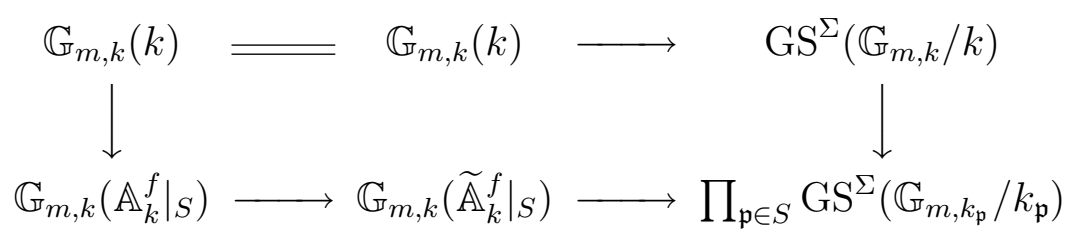


and the commutative diagram of modules

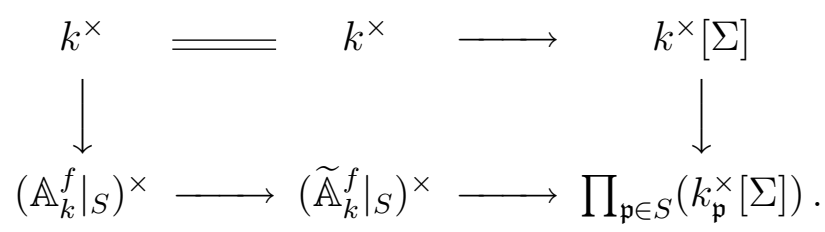

Proof. Assertion (i) follows immediately from the various definitions involved. Next, we verify assertion (ii). For a positive integer $n$, write $\mu_{n} \subseteq \bar{k}$ for the group of $n$-th roots of unity. Then, as is wellknown, for a positive $\Sigma$-integer $n$, there exist natural isomorphisms

$$
H^{1}\left(\Delta_{\mathbb{G}_{m, k} / k}^{\Sigma}, \mu_{n}\right) \stackrel{\sim}{\longrightarrow} H^{1}\left(\mathbb{G}_{m, \bar{k}}, \mu_{n}\right) \stackrel{\sim}{\longleftarrow}\left[u^{ \pm 1}\right]^{\times} /\left(\bar{k}\left[u^{ \pm 1}\right]^{\times}\right)^{n} .
$$

Thus, the invertible function $u \in \bar{k}\left[u^{ \pm 1}\right]^{\times}$determines an element of

$$
H^{1}\left(\Delta_{\mathbb{G}_{m, k} / k}^{\Sigma}, \widehat{\mathbb{Z}}^{\Sigma}(1)\right) \stackrel{\sim}{\longrightarrow} \operatorname{Hom}\left(\Delta_{\mathbb{G}_{m, k} / k}^{\Sigma}, \widehat{\mathbb{Z}}^{\Sigma}(1)\right) .
$$

On the other hand, one verifies easily that the resulting homomorphism $\Delta_{\mathbb{G}_{m, k} / k}^{\Sigma} \rightarrow \widehat{\mathbb{Z}}^{\Sigma}(1)$ is an isomorphism and satisfies the condition in the statement of assertion (ii). This completes the proof of assertion (ii). Assertion (iii) follows immediately from assertion (ii). This completes the proof of Lemma 3.2.

Lemma 3.3. The following hold:

(i) The exact sequence of modules

$$
1 \longrightarrow \mathfrak{o}_{k}^{\times} \longrightarrow k^{\times} \stackrel{\operatorname{div}_{k}}{\longrightarrow} \operatorname{Div}\left(\mathfrak{o}_{k}\right)
$$

determines an exact sequence of modules

$$
1 \longrightarrow \mathfrak{o}_{k}^{\times}[\Sigma] \longrightarrow k^{\times}[\Sigma] \stackrel{\operatorname{div}_{k}[\Sigma]}{\longrightarrow} \operatorname{Div}\left(\mathfrak{o}_{k}\right)[\Sigma] .
$$

(ii) There is no nontrivial element of the cokernel of the natural homomorphism $k^{\times} \rightarrow k^{\times}[\Sigma]$ which is annihilated by a $\Sigma$-integer.

Proof. First, we verify assertion (i). Write $M \stackrel{\text { def }}{=} \operatorname{Im}\left(\operatorname{div}_{k}\right) \subseteq \operatorname{Div}\left(\mathfrak{o}_{k}\right)$ for the image of $\operatorname{div}_{k}$. Then since $M$ is a free $\mathbb{Z}$-module, there exists a section of the natural surjection $k^{\times} \rightarrow M$; thus, we obtain a noncanonical isomorphism $\mathfrak{o}_{k}^{\times} \times M \stackrel{\sim}{\rightarrow} k^{\times}$. In particular, the natural homomorphism $\mathfrak{o}_{k}^{\times}[\Sigma] \rightarrow k^{\times}[\Sigma]$ is injective. Thus, to verify assertion (i), it suffices to verify that the kernel of $\operatorname{div}_{k}[\Sigma]$ is contained in $\mathfrak{o}_{k}^{\times}[\Sigma] \subseteq k^{\times}[\Sigma]$, or, equivalently [by the existence of the noncanonical isomorphism $\mathfrak{o}_{k}^{\times} \times M \stackrel{\sim}{\rightarrow} k^{\times}$, the natural homomorphism $M[\Sigma] \rightarrow$ $\operatorname{Div}\left(\mathfrak{o}_{k}\right)[\Sigma]$ is injective.

For an element $\sum a_{i} \cdot \mathfrak{p}_{i} \in \operatorname{Div}\left(\mathfrak{o}_{k}\right)$, where $a_{i} \in \mathbb{Z}$ and $\mathfrak{p}_{i} \in \mathfrak{P}_{k}^{f}$, write $\left[\sum a_{i} \cdot \mathfrak{p}_{i}\right] \in \operatorname{Pic}\left(\mathfrak{o}_{k}\right)=\operatorname{Div}\left(\mathfrak{o}_{k}\right) / M$ for the element of the cokernel of $\operatorname{div}_{k}$ determined by $\sum a_{i} \cdot \mathfrak{p}_{i} \in \operatorname{Div}\left(\mathfrak{o}_{k}\right)$. Now, for an element $a \in$ $\operatorname{Pic}\left(\mathfrak{o}_{k}\right)$, let us fix a nonarchimedean prime $\mathfrak{q}_{a} \in \mathfrak{P}_{k}^{f}$ of $k$ such that $a=$ $\left[1 \cdot \mathfrak{q}_{a}\right]$. [Note that it follows immediately from Chebotarev's density 
theorem that such a $\mathfrak{q}_{a}$ always exists.] Write $T \stackrel{\text { def }}{=}\left\{\mathfrak{q}_{a} \in \mathfrak{P}_{k}^{f} \mid a \in\right.$ $\left.\operatorname{Pic}\left(\mathfrak{o}_{k}\right)\right\}$. Moreover, for each $\mathfrak{p} \in \mathfrak{P}_{k}^{f} \backslash T$, write $x_{\mathfrak{p}} \stackrel{\text { def }}{=} 1 \cdot \mathfrak{p}-1 \cdot \mathfrak{q}_{[1 \cdot \mathfrak{p}]} \in$ $\operatorname{Div}\left(\mathfrak{o}_{k}\right)$. Then one verifies easily that the $\mathbb{Z}$-submodule $N \subseteq \operatorname{Div}\left(\mathfrak{o}_{k}\right)$ generated by $\left\{x_{\mathfrak{p}} \mid \mathfrak{p} \in \mathfrak{P}_{k}^{f} \backslash T\right\}$ is contained in $M$ and determines a section of the natural projection $\operatorname{Div}\left(\mathfrak{o}_{k}\right) \rightarrow \bigoplus_{\mathfrak{p} \in \mathfrak{P}_{k}^{f} \backslash T} \mathbb{Z}$. In particular, we obtain a commutative diagram of free $\mathbb{Z}$-modules

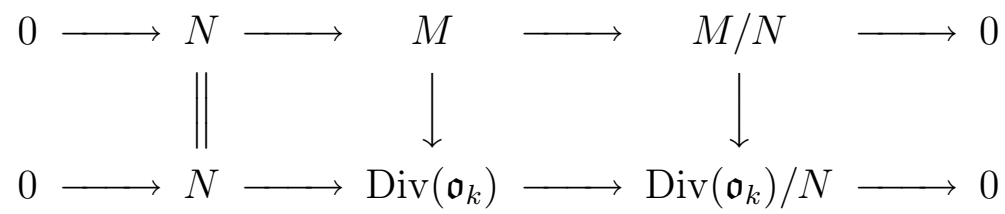

- where the horizontal sequences are exact, and the vertical arrows are injective. On the other hand, since $\operatorname{Pic}\left(\mathfrak{o}_{k}\right)$, hence also $T$, is finite, $M / N$ and $\operatorname{Div}\left(\mathfrak{o}_{k}\right) / N$ are finitely generated free $\mathbb{Z}$-modules. In particular, one verifies easily that the natural homomorphism $(M / N)[\Sigma] \rightarrow$ $\left(\operatorname{Div}\left(\mathfrak{o}_{k}\right) / N\right)[\Sigma]$ is injective. Thus, it follows immediately that the natural homomorphism in question $M[\Sigma] \rightarrow \operatorname{Div}\left(\mathfrak{o}_{k}\right)[\Sigma]$ is injective. This completes the proof of assertion (i).

Next, we verify assertion (ii). Now let us observe that one verifies easily that there is no nontrivial element of the cokernel of the natural homomorphism $\mathbb{Z} \rightarrow \widehat{\mathbb{Z}}^{\Sigma}$ which is annihilated by a $\Sigma$-integer. Thus, assertion (ii) follows immediately from the existence of the [noncanonical] isomorphism $\mathfrak{o}_{k}^{\times} \times M \stackrel{\sim}{\rightarrow} k^{\times}$obtained in the proof of assertion (i), together with the well-known fact that $\mathfrak{o}_{k}^{\times}$is finitely generated. This completes the proof of assertion (ii).

Remark 3.3.1. The observation given in the proof of Lemma 3.3 was related to the author by A. Tamagawa and $S$. Yasuda.

Lemma 3.4. By applying Lemma 3.2, (iii), let us identify $\mathbb{G}_{m, k}(k)$ (respectively, $\left.\mathbb{G}_{m, k}\left(\mathbb{A}_{k}^{f}\right) ; \operatorname{GS}^{\Sigma}\left(\mathbb{G}_{m, k} / k\right) ; \prod_{\mathfrak{p} \in \mathfrak{P}_{k}^{f}} \operatorname{GS}^{\Sigma}\left(\mathbb{G}_{m, k_{\mathfrak{p}}} / k_{\mathfrak{p}}\right)\right)$ with $k^{\times}$(respectively, $\left.\left(\mathbb{A}_{k}^{f}\right)^{\times} ; k^{\times}[\Sigma] ; \prod_{\mathfrak{p} \in \mathfrak{P}_{k}^{f}}\left(k_{\mathfrak{p}}^{\times}[\Sigma]\right)\right)$. Suppose that $k$ is either the field of rational numbers or an imaginary quadratic field. Let

$$
\begin{gathered}
\left(a_{\mathfrak{p}}\right)_{\mathfrak{p} \in \mathfrak{P}_{k}^{f}} \in\left(\mathbb{A}_{k}^{f}\right)^{\times} \simeq \mathbb{G}_{m, k}\left(\mathbb{A}_{k}^{f}\right) \\
a \in k^{\times}[\Sigma] \simeq \mathrm{GS}^{\Sigma}\left(\mathbb{G}_{m, k} / k\right)
\end{gathered}
$$

be such that their images in $\prod_{\mathfrak{p} \in \mathfrak{P}_{k}^{f}}\left(k_{\mathfrak{p}}^{\times}[\Sigma]\right) \simeq \prod_{\mathfrak{p} \in \mathfrak{P}_{k}^{f}} \mathrm{GS}^{\Sigma}\left(\mathbb{G}_{m, k_{\mathfrak{p}}} / k_{\mathfrak{p}}\right)[c f$. the diagrams of Lemma 3.2, (iii)] coincide. Then the following hold:

(i) $a^{d_{k}} \in k^{\times}[\Sigma] \simeq \mathrm{GS}^{\Sigma}\left(\mathbb{G}_{m, k} / k\right)$ is contained in the image of the natural homomorphism $k^{\times} \simeq \mathbb{G}_{m, k}(k) \rightarrow k^{\times}[\Sigma] \simeq \mathrm{GS}^{\Sigma}\left(\mathbb{G}_{m, k} / k\right)$.

(ii) If $d_{k}$ is a $\Sigma$-integer, then $a \in k^{\times}[\Sigma] \simeq \mathrm{GS}^{\Sigma}\left(\mathbb{G}_{m, k} / k\right)$ is contained in the image of the natural homomorphism $k^{\times} \simeq \mathbb{G}_{m, k}(k) \rightarrow$ $k^{\times}[\Sigma] \simeq \mathrm{GS}^{\Sigma}\left(\mathbb{G}_{m, k} / k\right)$. 
(iii) If $d_{k}$ is a $\Sigma$-integer, and we fix an element $\widetilde{a} \in k^{\times} \simeq \mathbb{G}_{m, k}(k)$ whose image in $k^{\times}[\Sigma] \simeq \operatorname{GS}^{\Sigma}\left(\mathbb{G}_{m, k} / k\right)$ coincides with a [cf. (ii)], then, for each $\mathfrak{p} \in \mathfrak{P}_{k}^{f}$ whose residue characteristic is $\in \Sigma$, the difference $a_{\mathfrak{p}} \cdot \widetilde{a}^{-1} \in k_{\mathfrak{p}}^{\times}$is a root of unity whose order is a (Primes $\left.\backslash \Sigma\right)$ integer.

Proof. First, we verify assertion (i). Since the image of $a \in k^{\times}[\Sigma] \simeq$ $\mathrm{GS}^{\Sigma}\left(\mathbb{G}_{m, k} / k\right)$ in $\prod_{\mathfrak{p} \in \mathfrak{P}_{k}^{f}}\left(k_{\mathfrak{p}}^{\times}[\Sigma]\right) \simeq \prod_{\mathfrak{p} \in \mathfrak{P}_{k}^{f}} \mathrm{GS}^{\Sigma}\left(\mathbb{G}_{m, k_{\mathfrak{p}}} / k_{\mathfrak{p}}\right)$ is contained in the image of the natural homomorphism $\left(\mathbb{A}_{k}^{f}\right)^{\times} \simeq \mathbb{G}_{m, k}\left(\mathbb{A}_{k}^{f}\right) \rightarrow$ $\prod_{\mathfrak{p} \in \mathfrak{P}_{k}^{f}}\left(k_{\mathfrak{p}}^{\times}[\Sigma]\right) \simeq \prod_{\mathfrak{p} \in \mathfrak{P}_{k}^{f}} \mathrm{GS}^{\Sigma}\left(\mathbb{G}_{m, k_{\mathfrak{p}}} / k_{\mathfrak{p}}\right)$, one verifies easily that the image of $a \in k^{\times}[\Sigma]$ by the homomorphism $\operatorname{div}_{k}[\Sigma]: k^{\times}[\Sigma] \rightarrow \operatorname{Div}\left(\mathfrak{o}_{k}\right)[\Sigma]$ is contain in the $\mathbb{Z}$-submodule $\operatorname{Div}\left(\mathfrak{o}_{k}\right) \subseteq \operatorname{Div}\left(\mathfrak{o}_{k}\right)[\Sigma]$. Thus, it follows immediately from the definition of $d_{k}$ that there exists $\widetilde{b} \in k^{\times}$such that the images $\widetilde{b}$ and $a^{d_{k}}$ in $\operatorname{Div}\left(\mathfrak{o}_{k}\right)[\Sigma]$ coincide. On the other hand, since $k$ is either the field of rational numbers or an imaginary quadratic field, it holds that $\mathfrak{o}_{k}^{\times}$is finite, which thus implies that $\mathfrak{o}_{k}^{\times} \rightarrow \mathfrak{o}_{k}^{\times}[\Sigma]$ is surjective. Thus, it follows immediately from Lemma 3.3, (i), that, by replacing $\widetilde{b}$ by a suitable element of $k^{\times}$, we conclude that $a^{d_{k}}$ coincides with the image of $\widetilde{b} \in k^{\times}$in $k^{\times}[\Sigma]$. This completes the proof of assertion (i).

Assertion (ii) follows immediately from Lemma 3.3, (ii), together with assertion (i); our assumption that $d_{k}$ is a $\Sigma$-integer. Finally, we verify assertion (iii). One verifies easily that, for each $\mathfrak{p} \in \mathfrak{P}_{k}^{f}$ whose residue characteristic is $\in \Sigma$, the kernel of the natural homomorphism $k_{\mathfrak{p}}^{\times} \rightarrow k_{\mathfrak{p}}^{\times}[\Sigma]$ consists of roots of unity in $k_{\mathfrak{p}}$ whose orders are $(\mathfrak{P r i m e s} \backslash \Sigma)$-integers. Thus, assertion (iii) follows immediately from assertion (ii). This completes the proof of assertion (iii).

Lemma 3.5. In the notation of Lemma 3.4, if $\Sigma=$ Primes, then the commutative diagram of sets

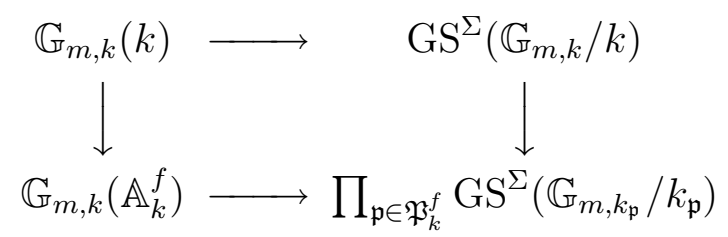

is cartesian.

Proof. This follows immediately from Lemma 3.4, (ii), (iii).

\section{CONDITIONAL RESUltS ON THE BIRATIONAL SECTION CONJECTURE}

In the present $\S 4$, we prove conditional results on the birational section conjecture for projective smooth curves over number fields. We maintain the notation of the preceding $\S 3$. 
First, let us recall a conditional result on the section conjecture over number fields. It seems to the author that [at least, a similar result to] the following result is likely to be well-known to experts. Since, however, the result could not be found in the literature, the author decided to give a proof.

Theorem 4.1. Let $\mathcal{C}$ be a full formation that contains all finite solvable groups, $k$ a number field [where we refer to the discussion entitled "Numbers" in $\S 0$ concerning the term "number field"], $X$ a projective smooth curve (respectively, hyperbolic curve) over $k$ [where we refer to the discussion entitled "Curves" in $§ 0$ concerning the term "smooth curve" (respectively, "hyperbolic curve")], and $s$ a pro-C birational Galois section (respectively, locally geometric pro-C Galois section) of $X / k[c f$. Definition 1.2 (respectively, Definitions $1.2 ; 2.1$ )]. Write $\mathfrak{P}_{k}^{f}$ for the set of nonarchimedean primes of $k$ and $X^{\mathrm{cpt}}$ for the [uniquely determined] smooth compactification of $X$ over $k$. For each $\mathfrak{p} \in \mathfrak{P}_{k}^{f}$, write $k_{\mathfrak{p}}$ for the $\mathfrak{p}$-adic completion of $k$. Then the following conditions are equivalent:

(1) $s$ is geometric [cf. Definition 1.3].

(2) There exist a subset $T \subseteq \mathfrak{P}_{k}^{f}$ of $\mathfrak{P}_{k}^{f}$ of density 0 and a closed subscheme $Z \subseteq X^{\mathrm{cpt}}$ of $X^{\mathrm{cpt}}$ which is finite over $k$ such that, for each $\mathfrak{p} \in \mathfrak{P}_{k}^{f} \backslash T$, the [image of the uniquely determined - cf. Lemma 2.7] $k_{\mathfrak{p}}$-valued point of $X^{\mathrm{cpt}}$ associated to $s$ [cf. Definition 2.6; Proposition 2.9 (respectively, Definition 2.3)] is contained in $Z \subseteq X^{\mathrm{cpt}}$.

Proof. First, we verify Theorem 4.1 in the case where $s$ is a locally geometric pro-C Galois section. The implication $(1) \Rightarrow(2)$ is immediate [cf. also Remark 2.3.1]. Next, we verify the implication $(2) \Rightarrow(1)$. Now observe that it follows from the equivalence $(1) \Leftrightarrow(2)$ of Lemma 1.6 that, to verify the implication $(2) \Rightarrow(1)$, by replacing $k$ by a suitable finite extension of $k$, we may assume without loss of generality that $k$ is totally imaginary. Next, observe that, for each open subgroup $H \subseteq \Pi_{X / k}^{\mathcal{C}}$ of $\Pi_{X / k}^{\mathcal{C}}$ containing the image of $s$, if we write $Y$ for the connected finite étale covering of $X$ corresponding to $H \subseteq \Pi_{X / k}^{\mathcal{C}}$ [thus, $\Pi_{Y / k}^{\mathcal{C}}=H \subseteq \Pi_{X / k}^{\mathcal{C}}$ ], then since the morphism $Y \rightarrow X$ is finite, one verifies easily that the pro- $\mathcal{C}$ Galois section of $Y / k$ naturally determined by $s$ [which is necessarily locally geometric by the various definitions involved] satisfies condition (2). Thus, to verify the implication $(2) \Rightarrow(1)$, by replacing $X$ by such a suitable $Y$, we may assume without loss of generality that $X$ is of genus $\geq 2$; moreover, it follows from the equivalence $(1) \Leftrightarrow(3)$ of Lemma 1.6 that, to verify the implication $(2) \Rightarrow(1)$, by applying the conclusion to various open subgroups of $\Pi_{X / k}^{\mathcal{C}}$ containg the image of $s$, it suffices to verify that $X^{\mathrm{cpt}}(k) \neq \emptyset$. In particular, since $X$ is of genus $\geq 2$, and [one verifies easily that] the pro- $\mathcal{C}$ Galois section of $X^{\mathrm{cpt}} / k$ naturally determined by $s$ is locally geometric and satisfies condition (2), to verify 
that $X^{\mathrm{cpt}}(k) \neq \emptyset$, by replacing $X$ by $X^{\mathrm{cpt}}$, we may assume without loss of generality that $X^{\mathrm{cpt}}=X$.

Now since $s$ is locally geometric, and $k$ is totally imaginary, it follows

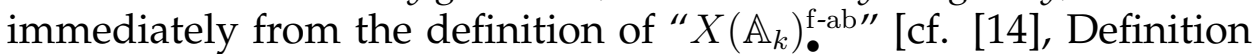
5.4 , (3)] that the [uniquely determined] $k_{\mathfrak{p}}$-valued points of $X$ associated to $s-$ where $\mathfrak{p}$ ranges over nonarchimedean primes of $k-$ form a part of an element of $X\left(\mathbb{A}_{k}\right)_{\bullet}^{\text {f-ab }}$. Thus, it follows immediately from [14], Theorem 8.2, together with condition (2), that $Z(k) \neq \emptyset$, hence also $X(k) \neq \emptyset$. This completes the proof of the implication $(2) \Rightarrow(1)$, hence also of Theorem 4.1 in the case where $s$ is a locally geometric pro-C Galois section.

Next, we verify Theorem 4.1 in the case where $s$ is a pro-C $\mathcal{C}$ birational Galois section. The implication $(1) \Rightarrow(2)$ is immediate [cf. also Remark 2.6.1]. Next, we verify the implication $(2) \Rightarrow(1)$. First, observe that it follows immediately from a similar argument to the argument applied in the proof of Theorem 4.1 in the case where $s$ is a locally geometric pro- $\mathcal{C}$ Galois section that, to verify Theorem 4.1 in the case where $s$ is a pro-C birational Galois section, by replacing $\Pi_{k(X) / k}^{\mathcal{C}}$ by an open subgroup of $\Pi_{k(X) / k}^{\mathcal{C}}$ containing the image of $s$, we may assume without loss of generality that $X$ is of genus $\geq 2$; moreover, it follows from the equivalence $(1) \Leftrightarrow(3)$ of Lemma 1.5 that, to verify the implication $(2) \Rightarrow(1)$, by applying the conclusion to various open subgroups of $\Pi_{k(X) / k}^{\mathcal{C}}$ containg the image of $s$, it suffices to verify that $X(k) \neq \emptyset$. On the other hand, since $X$ is of genus $\geq 2$, in light of Proposition 2.11, by applying Theorem 4.1 in the case where $s$ is a locally geometric pro- $\mathcal{C}$ Galois section to the pro- $\mathcal{C}$ Galois section of $X / k$ naturally determined by $s$, we conclude that $X(k) \neq \emptyset$. This completes the proof of the implication $(2) \Rightarrow(1)$, hence also of Theorem 4.1 in the case where $s$ is a pro-C birational Galois section.

Corollary 4.2. Let $\mathcal{C}$ be a full formation that contains all finite solvable groups, $k$ a number field [where we refer to the discussion entitled "Numbers" in $§ 0$ concerning the term "number field"], and $X$ a projective smooth curve (respectively, hyperbolic curve) over $k$ [where we refer to the discussion entitled "Curves" in $§ 0$ concerning the term "smooth curve" (respectively, "hyperbolic curve")]. Suppose that there exist an abelian variety $A$ over $k$ and a nonconstant morphism $X \rightarrow A$ over $k$ such that both the Mordell-Weil group and the Shafarevich-Tate group of $A / k$ are finite. Then any pro-C birational Galois section (respectively, any locally geometric pro-C Galois section) of $X / k[c f$. Definition 1.2 (respectively, Definitions 1.2; 2.1)] is geometric [cf. Definition 1.3].

Proof. Write

$$
\widehat{\operatorname{Sel}}^{f}(A / k) \stackrel{\text { def }}{=}{\underset{n}{n}}_{\lim } \operatorname{Ker}\left(H^{1}(k, A(\bar{k})[n]) \rightarrow \prod_{\mathfrak{p} \in \mathfrak{P}_{k}^{f}} H^{1}\left(k_{\mathfrak{p}}, A(\bar{k})\right)\right)
$$


- where the projective limit is over all positive integers $n$, and $A(\bar{k})[n]$ is the subgroup of $A(\bar{k})$ consisting of elements of $A(\bar{k})$ that are annihilated by $n$. Then the well-known natural $G_{k}$-equivariant isomorphism $A(\bar{k})[n] \stackrel{\sim}{\rightarrow} \Delta_{A / k}^{\mathfrak{P r i m} \mathfrak{r} \mathfrak{s}} / n \Delta_{A / k}^{\mathfrak{P r i m e s}}$ induces a natural injection $\widehat{\operatorname{Sel}}^{f}(A / k) \hookrightarrow H^{1}\left(k, \Delta_{A / k}^{\mathfrak{P r i m} e \mathfrak{g}}\right)$; moreover, it follows immediately from the various definitions involved that the pro-Primes Kummer homomorphism $A(k) \rightarrow H^{1}\left(k, \Delta_{A / k}^{\mathfrak{P r i m e g}}\right)$ associated to $A$ [cf., e.g., [6], Remark 1.1.4, (iii)] factors through $\widehat{\operatorname{Sel}}^{f}(A / k) \subseteq H^{1}\left(k, \Delta_{A / k}^{\mathfrak{B r i m e g}}\right)$, which thus implies that we have a natural injection $A(k) \hookrightarrow \widehat{\operatorname{Sel}}^{f}(A / k)$. [Here, this injectivity is a formal consequence of the well-known fact that there is no nontrivial divisible element of $A(k)$.] On the other hand, since the Shafarevich-Tate group of $A / k$ is finite, in light of the fact that the absolute Galois group of the completion of $k$ at an archimedean prime is either $\simeq \mathbb{Z} / 2 \mathbb{Z}$ or $\simeq\{1\}$, one verifies easily that, for each positive integer $n$, the cokernel of the natural homomorphism

$$
A(k) / n A(k) \longrightarrow \operatorname{Ker}\left(H^{1}(k, A(\bar{k})[n]) \rightarrow \prod_{\mathfrak{p} \in \mathfrak{P}_{k}^{f}} H^{1}\left(k_{\mathfrak{p}}, A(\bar{k})\right)\right)
$$

is annihilated by a positive integer which does not depend on $n$. Thus, since the Mordell-Weil group of $A / k$ is finite, it follows immediately that the resulting injection $A(k) \hookrightarrow \widehat{\operatorname{Sel}}^{f}(A / k)$ is an isomorphism.

Let $s$ be a pro- $\mathcal{C}$ birational Galois section (respectively, locally geometric pro-C Galois section) of $X / k$. Write $s^{A}$ for the pro-Primes Galois section of $A / k$ obtained as the composite

$$
\begin{gathered}
G_{k} \stackrel{s}{\longrightarrow} \Pi_{k(X) / k}^{\mathcal{C}} \longrightarrow \Pi_{X / k}^{\mathcal{C}} \longrightarrow \Pi_{A / k}^{\mathcal{C}}=\Pi_{A / k}^{\mathfrak{P r i m e s}} \\
\text { (respectively, } G_{k} \stackrel{s}{\longrightarrow} \Pi_{X / k}^{\mathcal{C}} \longrightarrow \Pi_{A / k}^{\mathcal{C}}=\Pi_{A / k}^{\mathfrak{P r i m e s}} \text { ) }
\end{gathered}
$$

- where the third (respectively, second) arrow is the homomorphism over $G_{k}$ induced by the nonconstant morphism $X \rightarrow A$ over $k$. Then $s^{A}$ naturally determines an element of $H^{1}\left(k, \Delta_{A / k}^{\mathfrak{P r i m e q}}\right)$ [cf., e.g., [6], Remark 1.1.4, (ii)]; moreover, it follows immediately from Proposition 2.9 (respectively, our assumption that $s$ is locally geometric), together with the various definitions involved, that this element is contained in $A(k) \stackrel{\sim}{\rightarrow} \widehat{\operatorname{Sel}}^{f}(A / k) \subseteq H^{1}\left(k, \Delta_{A / k}^{\mathfrak{B r i m} e \mathfrak{g}}\right)$. In particular, since $X \rightarrow A$ is nonconstant, and the Mordell-Weil group $A(k)$ is finite, it follows immediately from the injectivity of the pro-Primes Kummer homomorphism associated to $A \otimes_{k} k_{\mathfrak{p}}$ [that is a formal consequence of the well-known fact that there is no nontrivial divisible element of $A\left(k_{\mathfrak{p}}\right)$ ], together with [6], Remark 1.1.4, (iii), that $s$ satisfies condition (2) of Theorem 4.1. Thus, it follows from the implication (2) $\Rightarrow$ (1) 
of Theorem 4.1 that $s$ is geometric. This completes the proof of Corollary 4.2 .

Remark 4.2.1. As in the case of [4], Theorem 17, one may apply Corollary 4.2 to obtain some examples of projective smooth curves over number fields for which any prosolvable birational Galois section [i.e., any pro- $\mathcal{C}$ birational Galois section in the case where $\mathcal{C}$ consists of all finite solvable groups] is geometric [cf., e.g., the discussions in [4], Remark 18, (1); [14], Example 8.7; [14], Corollary 8.8].

Remark 4.2.2. The observation given in the proof of Corollary 4.2 was related to the author by $A$. Tamagawa and $S$. Yasuda.

Definition 4.3. Suppose that $X$ is a hyperbolic curve over [the number field] $k$. Let $s$ be a pro-C Galois section of $X / k$ [cf. Definition 1.2].

(i) We shall say that $s$ is cuspidal if the image of $s$ is contained in a decomposition subgroup of $\Pi_{X / k}^{\mathcal{C}}$ associated to a cusp of $X / k$.

(ii) We shall say that $s$ is unramified almost everywhere if the composite

$$
G_{k} \stackrel{s}{\longrightarrow} \Pi_{X / k}^{\mathcal{C}} \longrightarrow \operatorname{Aut}\left(\Delta_{X / k}^{\mathcal{C}}\right)
$$

- where the second arrow is the action of $\Pi_{X / k}^{\mathcal{C}}$ on $\Delta_{X / k}^{\mathcal{C}}$ obtained by conjugation - is unramified for all but finitely many $\mathfrak{p} \in \mathfrak{P}_{k}^{f}$.

Remark 4.3.1. In the notation of Definition 4.3, it is immediate that if $s$ is cuspidal [cf. Definition 4.3, (i)], then $s$ is geometric [cf. Definition 1.3].

Proposition 4.4. Suppose that $\Sigma$ is finite. Then any geometric [cf. Definition 1.3] pro- $\Sigma$ Galois section [cf. Definition 1.2] of a hyperbolic curve over a number field is either cuspidal [cf. Definition 4.3, (i)] or unramified almost everywhere [cf. Definition 4.3, (ii)].

Proof. This follows immediately from Proposition A.7.

Next, we prove the main result of the present paper.

Theorem 4.5. Let $\mathcal{C}$ be a full formation, $k$ either the field of rational numbers or an imaginary quadratic field, $X$ a projective smooth curve over $k$ [where we refer to the discussion entitled "Curves" in $\S 0$ concerning the term "smooth curve"], and s a pro-C birational Galois section of $X / k$ [cf. Definition 1.2]. Write $\mathfrak{o}_{k}$ for the ring of integers of $k$ and $\mathfrak{P}_{k}^{f}$ for the set of nonarchimedean primes of $k$. For each $\mathfrak{p} \in \mathfrak{P}_{k}^{f}$, write $k_{\mathfrak{p}}$ for the $\mathfrak{p}$-adic completion of $k$ and $\mathfrak{o}_{\mathfrak{p}}$ for the ring of integers of $k_{\mathfrak{p}}$. Write, moreover, $\mathbb{A}_{k}^{f}$ for the finite part of the adele ring of $k$, i.e.,

$$
\mathbb{A}_{k}^{f} \stackrel{\text { def }}{=}\left\{\left(a_{\mathfrak{p}}\right)_{\mathfrak{p} \in \mathfrak{P}_{k}^{f}} \in \prod_{\mathfrak{p} \in \mathfrak{P}_{k}^{f}} k_{\mathfrak{p}} \mid a_{\mathfrak{p}} \in \mathfrak{o}_{\mathfrak{p}} \text { for all but finitely many } \mathfrak{p}\right\} .
$$

Suppose that the following three conditions are satisfied: 
(a) The pro-C birational Galois section $s$ is locally geometric [cf. Definition 2.5].

(b) $\Sigma(\mathcal{C})$ [where we refer to the discussion entitled "Profinite groups" in $\S 0$ concerning the set $\Sigma(\mathcal{C})]$ is cofinite, i.e., Primes $\backslash \Sigma(\mathcal{C})$ [where we refer to the discussion entitled "Numbers" in $§ 0$ concerning the set $\mathfrak{P r i m e s}]$ is finite.

(c) $\operatorname{Pic}\left(\mathfrak{o}_{k}\right) \stackrel{\text { def }}{=} \operatorname{Pic}\left(\operatorname{Spec} \mathfrak{o}_{k}\right)$ is annihilated by a $\Sigma(\mathcal{C})$-integer [where we refer to the discussion entitled "Numbers" in $\S 0$ concerning the term " $\Sigma(\mathcal{C})$-integer"].

[Note that it follows from Proposition 2.9 that if $\Sigma(\mathcal{C})=\mathfrak{P r i m e s}$, or, equivalently [cf. the discussion entitled "Profinite groups" in $\S 0], \mathcal{C}$ contains all finite solvable groups, then the above three conditions are satisfied.] Then the following conditions are equivalent:

(1) The pro-C birational Galois section $s$ is geometric $[c f$. Definition 1.3].

(2) The following two conditions are satisfied:

(2-i) There exist a finite morphism $\phi: X \rightarrow \mathbb{P}_{k}^{1}$ over $k$ and, for each $\mathfrak{p} \in \mathfrak{P}_{k}^{f}$, a $k_{\mathfrak{p}}$-valued point $x_{\mathfrak{p}}$ of $X$ associated to $s[c f$. Definition 2.6; condition (a)] [note that if the residue characteristic of $\mathfrak{p}$ is $\in \Sigma(\mathcal{C})$, then the $k_{\mathfrak{p}}$-valued point $x_{\mathfrak{p}}$ of $X$ associated to $s$ is uniquely determined - cf. Lemma 2.7] such that the composite

$$
\text { Spec } k_{\mathfrak{p}} \stackrel{x_{\mathfrak{p}}}{\longrightarrow} X \stackrel{\phi}{\longrightarrow} \mathbb{P}_{k}^{1}
$$

determines a $k_{\mathfrak{p}}$-valued point of $\mathbb{P}_{k}^{1} \backslash\{0,1, \infty\}$.

(2-ii) For each open subscheme $U \subseteq X$ of $X$ which is a hyperbolic curve over $k$ where we refer to the discussion entitled "Curves" in $\S 0$ concerning the term "hyperbolic curve"], there exists a prime number $l_{U} \in \Sigma(\mathcal{C})$ contained in $\Sigma(\mathcal{C})$ such that the pro- $l_{U}$ Galois section of $U / k[c f$. Definition 1.2] naturally determined by $s$ is either cuspidal [cf. Definition 4.3, (i)] or unramified almost everywhere [cf. Definition 4.3, (ii)].

(3) There exist a finite morphism $\phi: X \rightarrow \mathbb{P}_{k}^{1}$ over $k$ and an $\mathbb{A}_{k}^{f}$-valued point $x_{\mathbb{A}}$ of $X$ associated to $s$ [cf. Definition 2.6; condition (a)] $\left[\right.$ note that if $\Sigma(\mathcal{C})=\mathfrak{P r i m e s}$, then the $\mathbb{A}_{k}^{f}$-valued point $x_{\mathbb{A}}$ of $X$ associated to $s$ is uniquely determined - cf. Lemma 2.7] such that the composite

$$
\operatorname{Spec} \mathbb{A}_{k}^{f} \stackrel{x_{\mathbb{A}}}{\longrightarrow} X \stackrel{\phi}{\longrightarrow} \mathbb{P}_{k}^{1}
$$

determines an $\mathbb{A}_{k}^{f}$-valued point of $\mathbb{P}_{k}^{1} \backslash\{0,1, \infty\} \subseteq \mathbb{P}_{k}^{1}$.

(4) There exist a finite subset $T \subseteq \mathfrak{P}_{k}^{f}$ of $\mathfrak{P}_{k}^{f}$ and a closed subscheme $Z \subseteq X$ of $X$ which is finite over $k$ such that, for each $\mathfrak{p} \in \mathfrak{P}_{k}^{f} \backslash T$ whose residue characteristic is $\in \Sigma(\mathcal{C})$, the [image of the uniquely 
determined - cf. Lemma 2.7] $k_{\mathfrak{p}}$-valued point $x_{\mathfrak{p}}$ of $X$ associated to $s$ [cf. Definition 2.6; condition (a)] is contained in $Z \subseteq X$.

Proof. The implication (1) $\Rightarrow(2)$ follows immediately from Proposition 4.4, together with Remark 2.6.1. Next, we verify the implication $(2) \Rightarrow(3)$. Suppose that condition (2) is satisfied. Then, by condition (2-i), for each $\mathfrak{p} \in \mathfrak{P}_{k}^{f}$, the composite

$$
\text { Spec } k_{\mathfrak{p}} \stackrel{x_{\mathfrak{p}}}{\longrightarrow} X \stackrel{\phi}{\longrightarrow} \mathbb{P}_{k}^{1}
$$

determines a $k_{\mathfrak{p}}$-valued point of $\mathbb{P}_{k}^{1} \backslash\{0,1, \infty\}$. Thus, to verify the implication $(2) \Rightarrow(3)$, it suffices to verify that the above $k_{\mathfrak{p}}$-valued point of $\mathbb{P}_{k}^{1} \backslash\{0,1, \infty\}$ obtained as the composite $\phi \circ x_{\mathfrak{p}}$ determines an $\mathfrak{o}_{\mathfrak{p}}$-valued point of $\mathbb{P}_{\mathfrak{o}_{\mathfrak{p}}}^{1} \backslash\{0,1, \infty\}$ for all but finitely many $\mathfrak{p} \in \mathfrak{P}_{k}^{f}$. Write $U \subseteq X$ for the open subscheme of $X$ obtained as the inverse image of $\mathbb{P}_{k}^{1} \backslash\{0,1, \infty\} \subseteq \mathbb{P}_{k}^{1}$ by $\phi$. Then, by condition (2-ii), there exists a prime number $l_{U} \in \Sigma(\mathcal{C})$ contained in $\Sigma(\mathcal{C})$ such that the pro- $l_{U}$ Galois section $s^{U}$ of $U / k$ obtained as the composite

$$
G_{k} \stackrel{s}{\longrightarrow} \Pi_{k(X) / k}^{\mathcal{C}} \longrightarrow \Pi_{U / k}^{\left\{l_{U}\right\}}
$$

is either cuspidal or unramified almost everywhere. Write $s^{\mathbb{P}}$ for the pro$l_{U}$ Galois section of $\mathbb{P}_{k}^{1} \backslash\{0,1, \infty\}$ obtained as the composite

$$
G_{k} \stackrel{s}{\longrightarrow} \Pi_{k(X) / k}^{\mathcal{C}} \longrightarrow \Pi_{U / k}^{\left\{l_{U}\right\}} \longrightarrow \prod_{\left(\mathbb{P}_{k}^{1} \backslash\{0,1, \infty\}\right) / k}^{\left\{l_{l}\right\}}
$$

- where the third arrow is the homomorphism over $G_{k}$ induced by $\phi$. Then since the morphism $U \rightarrow \mathbb{P}_{k}^{1} \backslash\{0,1, \infty\}$ induced by $\phi$ is finite, one verifies easily that the homomorphism $\Pi_{U / k}^{\left\{l_{U}\right\}} \rightarrow \Pi_{\left(\mathbb{P}_{k}^{1} \backslash\{0,1, \infty\}\right) / k}^{\left\{l_{U}\right\}}$ maps injectively any cuspidal decomposition subgroup of $\Pi_{U / k}^{\left\{l_{U}\right\}}$ associated to a cusp of $U / k$ to a cuspidal decomposition subgroup of $\Pi_{\left(\mathbb{P}_{k}^{1} \backslash\{0,1, \infty\}\right) / k}^{\left\{l_{l}\right\}}$ associated to a cusp of $\mathbb{P}_{k}^{1} \backslash\{0,1, \infty\}$. Thus, it follows immediately that if $s^{U}$ is cuspidal, then $s^{\mathbb{P}}$ is cuspidal. On the other hand, by applying Lemma 1.4 [to " $\phi \circ x_{\mathfrak{p}}$ " for $\mathfrak{p} \in \mathfrak{P}_{k}^{f}$ whose residue characteristic is $=l_{U}$ ], it follows immediately from condition (2-i) that $s^{\mathbb{P}}$ is not cuspidal. Thus, we conclude that $s^{U}$ is not cuspidal, hence also [by condition (2-ii)] unramified almost everywhere. In particular, it follows Proposition A.10, (ii), that $s^{\mathbb{P}}$ is unramified almost everywhere. Therefore, it follows immediately from Proposition A.7, together with condition (2-i), that the $k_{\mathfrak{p}}$-valued point of $\mathbb{P}_{k}^{1}$ obtained as the composite $\phi \circ x_{\mathfrak{p}}$ determines an $\mathfrak{o}_{\mathfrak{p}}$-valued point of $\mathbb{P}_{\mathfrak{o}_{\mathfrak{p}}}^{1} \backslash\{0,1, \infty\}$ for all but finitely many $\mathfrak{p} \in \mathfrak{P}_{k}^{f}$. This completes the proof of the implication $(2) \Rightarrow(3)$.

Next, we verify the implication $(3) \Rightarrow(4)$. Suppose that condition (3) is satisfied. Write $s^{\mathbb{G}}$ for the pro- $\Sigma(\mathcal{C})$ Galois section of $\mathbb{G}_{m, k} \stackrel{\text { def }}{=}$ 
$\mathbb{P}_{k}^{1} \backslash\{0, \infty\}$ over $k$ obtained as the composite

$$
G_{k} \stackrel{s}{\rightarrow} \prod_{k(X) / k}^{\mathcal{C}} \rightarrow \prod_{k\left(\mathbb{P}_{k}^{1}\right) / k}^{\mathcal{C}} \rightarrow \prod_{\mathbb{G}_{m, k} / k}^{\mathcal{C}}=\Pi_{\mathbb{G}_{m, k} / k}^{\Sigma(\mathcal{C})}
$$

- where the second arrow is the homomorphism over $G_{k}$ induced by $\phi-$ and $t^{\mathbb{G}}$ for the pro- $\Sigma(\mathcal{C})$ Galois section of $\mathbb{G}_{m, k}$ over $k$ obtained as the composite

$$
G_{k} \stackrel{s}{\rightarrow} \Pi_{k(X) / k}^{\mathcal{C}} \rightarrow \Pi_{k\left(\mathbb{P}_{k}^{1}\right) / k}^{\mathcal{C}} \stackrel{\sim}{\rightarrow} \Pi_{k\left(\mathbb{P}_{k}^{1}\right) / k}^{\mathcal{C}} \rightarrow \prod_{\mathbb{G}_{m, k} / k}^{\mathcal{C}}=\Pi_{\mathbb{G}_{m, k} / k}^{\Sigma(\mathcal{C})}
$$

- where the second arrow is the homomorphism over $G_{k}$ induced by $\phi$, and the third arrow is the automorphism over $G_{k}$ induced by the automorphism of $\mathbb{P}_{k}^{1}$ over $k$ given by " $u \mapsto 1-u$ ". Then it follows immediately from condition (3) that there exists an element $\left(a_{\mathfrak{p}}\right)_{\mathfrak{p} \in \mathfrak{P}_{k}^{f}} \in\left(\widetilde{\mathbb{A}}_{k}^{f}\right)^{\times}$such that $\left(a_{\mathfrak{p}}\right)_{\mathfrak{p} \in \mathfrak{P}_{k}^{f}},\left(1-a_{\mathfrak{p}}\right)_{\mathfrak{p} \in \mathfrak{P}_{k}^{f}} \in\left(\mathbb{A}_{k}^{f}\right)^{\times} \simeq$ $\mathbb{G}_{m, k}\left(\mathbb{A}_{k}^{f}\right)$, and, moreover, the respective images of the pro- $\Sigma(\mathcal{C}) \mathrm{Ga}$ lois sections $s^{\mathbb{G}}, t^{\mathbb{G}} \in \mathrm{GS}^{\Sigma(\mathcal{C})}\left(\mathbb{G}_{m, k} / k\right) \simeq k^{\times}[\Sigma(\mathcal{C})]$ [cf. Lemma 3.2, (i)] in the set $\prod_{\mathfrak{p} \in \mathfrak{P}_{k}^{f}} \mathrm{GS}^{\Sigma(\mathcal{C})}\left(\mathbb{G}_{m, k_{\mathfrak{p}}} / k_{\mathfrak{p}}\right) \simeq \prod_{\mathfrak{p} \in \mathfrak{P}_{k}^{f}}\left(k_{\mathfrak{p}}^{\times}[\Sigma(\mathcal{C})]\right)$ [cf. the diagrams of Lemma 3.2, (iii)] coincide with the respective images of the elements $\left(a_{\mathfrak{p}}\right)_{\mathfrak{p} \in \mathfrak{P}_{k}^{f}},\left(1-a_{\mathfrak{p}}\right)_{\mathfrak{p} \in \mathfrak{P}_{k}^{f}} \in\left(\mathbb{A}_{k}^{f}\right)^{\times} \simeq \mathbb{G}_{m, k}\left(\mathbb{A}_{k}^{f}\right)$ in the set $\prod_{\mathfrak{p} \in \mathfrak{P}_{k}^{f}}\left(k_{\mathfrak{p}}^{\times}[\Sigma(\mathcal{C})]\right) \simeq \prod_{\mathfrak{p} \in \mathfrak{P}_{k}^{f}} \operatorname{GS}^{\Sigma(\mathcal{C})}\left(\mathbb{G}_{m, k_{\mathfrak{p}}} / k_{\mathfrak{p}}\right)$. Thus, it follows from Lemma 3.4, (ii), (iii); together with condition (c), that

(*): there exist $\widetilde{a}_{s}, \widetilde{a}_{t} \in k^{\times}$such that, for $\mathfrak{p} \in \mathfrak{P}_{k}^{f}$, if we write $u_{\mathfrak{p}} \stackrel{\text { def }}{=} a_{\mathfrak{p}} \cdot \widetilde{a}_{s}^{-1}, v_{\mathfrak{p}} \stackrel{\text { def }}{=}\left(1-a_{\mathfrak{p}}\right) \cdot \widetilde{a}_{t}^{-1} \in k_{\mathfrak{p}}^{\times}$, and the residue characteristic of $\mathfrak{p}$ is $\in \Sigma(\mathcal{C})$, then $u_{\mathfrak{p}}, v_{\mathfrak{p}}$ are roots of unity of $k_{\mathfrak{p}}$ whose orders are $(\mathfrak{P r i m e s} \backslash \Sigma(\mathcal{C}))$-integers.

Now let us observe that, for $\mathfrak{p} \in \mathfrak{P}_{k}^{f}$, the pair $\left(u_{\mathfrak{p}}, v_{\mathfrak{p}}\right)$ satisfies the equation

$$
1=\widetilde{a}_{s} \cdot u_{\mathfrak{p}}+\widetilde{a}_{t} \cdot v_{\mathfrak{p}} .
$$

Thus, it follows immediately from [2], Theorem 1.1, together with condition (b), that the set $\left\{\left(u_{\mathfrak{p}}, v_{\mathfrak{p}}\right)\right\}_{\mathfrak{p} \in \mathfrak{P}_{k}^{f}}$, hence also the set $\left\{u_{\mathfrak{p}}\right\}_{\mathfrak{p} \in \mathfrak{P}_{k}^{f}}$, is finite. In particular, since $a_{\mathfrak{p}}=\widetilde{a}_{s} \cdot u_{\mathfrak{p}}$ [cf. $\left.(*)\right]$, it follows immediately that the pro- $\mathcal{C}$ birational Galois section of $\mathbb{P}_{k}^{1} / k$ obtained as the composite

$$
G_{k} \stackrel{s}{\longrightarrow} \prod_{k(X) / k}^{\mathcal{C}} \longrightarrow \Pi_{k\left(\mathbb{P}_{k}^{1}\right) / k}^{\mathcal{C}}
$$

- where the second arrow is the homomorphism over $G_{k}$ induced by $\phi$ - satisfies condition (4). Therefore, since $\phi$ is finite, one verifies easily that the pro- $\mathcal{C}$ birational Galois section $s$ satisfies condition (4). This completes the proof of the implication $(3) \Rightarrow(4)$.

Finally, we verify the implication $(4) \Rightarrow(1)$. Suppose that condition (4) is satisfied. Let us fix an element $\mathfrak{p}_{0} \in \mathfrak{P}_{k}^{f} \backslash T$ of $\mathfrak{P}_{k}^{f} \backslash T$ such that the residue characteristic of $\mathfrak{p}_{0}$ is $\in \Sigma(\mathcal{C})$ [note that, by condition (b), such a $\mathfrak{p}_{0}$ always exists] and write $r\left(\mathfrak{p}_{0}\right)$ for the cardinality of 
the set of roots of unity of $k_{\mathrm{p}_{0}}$. Now observe that, for any open subgroup $H \subseteq \Pi_{k(X) / k}^{\mathcal{C}}$ of $\Pi_{k(X) / k}^{\mathcal{C}}$ containing the image of $s$, if we write $Y$ for the normalization of $X$ in the finite extension of $k(X)$ corresponding to $H \subseteq \Pi_{k(X) / k}^{\mathcal{C}}$ [thus, $\Pi_{k(Y) / k}^{\mathcal{C}}=H \subseteq \Pi_{k(X) / k}^{\mathcal{C}}$, then since the morphism $Y \rightarrow X$ is finite, the pro- $\mathcal{C}$ birational Galois section of $Y / k$ determined by $s$ satisfies condition (4) relative to the finite subset " $T$ " $\subseteq \mathfrak{P}_{k}^{f}$ appearing in condition (4). Thus, it follows from the equivalence $(1) \Leftrightarrow(3)$ of Lemma 1.5 that, to verify condition (1), by applying the conclusion to various such $H^{\prime}$ 's, it suffices to verify that $X$ admits a $k\left(\zeta_{r\left(\mathfrak{p}_{0}\right)}\right)$-valued point - where we use the notation $\zeta_{r\left(\mathfrak{p}_{0}\right)} \in \bar{k}$ to denote a primitive $r\left(\mathfrak{p}_{0}\right)$-th root of unity.

For each $\mathfrak{p} \in \mathfrak{P}_{k}^{f}$, let us fix a $k_{\mathfrak{p}}$-valued point $x_{\mathfrak{p}}$ of $X$ associated to $s$ [cf. condition (a)]. Now since $X$ is projective, there exists a closed immersion $X \hookrightarrow \mathbb{P}_{k}^{N}$ over $k$ for some positive integer $N$. Then it follows immediately from condition (4) that there exists a hyperplane $H \subseteq$ $\mathbb{P}_{k}^{N}$ defined over $k$ such that, for any $\mathfrak{p} \in \mathfrak{P}_{k}^{f}$, [the image of] the fixed $k_{\mathfrak{p}}$-valued point $x_{\mathfrak{p}}$ of $X$ is contained in $X \backslash(X \cap H) \subseteq \mathbb{P}_{k}^{N} \backslash H \simeq \mathbb{A}_{k}^{N}$. Moreover, again by condition (4) - by considering a suitable automorphism of $\mathbb{A}_{k}^{1}$ over $k$ - we may assume without loss of generality that, for each $i \in\{1, \cdots, N\}$ and $\mathfrak{p} \in \mathfrak{P}_{k}^{f}$, the $k_{\mathfrak{p}}$-valued point of $\mathbb{A}_{k}^{1}$ obtained as the composite

$$
\text { Spec } k_{\mathfrak{p}} \stackrel{x_{\mathfrak{p}}}{\rightarrow} X \backslash(X \cap H) \hookrightarrow \mathbb{P}_{k}^{N} \backslash H \simeq \mathbb{A}_{k}^{N} \stackrel{\mathrm{pr}_{i}}{\rightarrow} \mathbb{A}_{k}^{1}
$$

factors through $\mathbb{G}_{m, k} \stackrel{\text { def }}{=} \mathbb{A}_{k}^{1} \backslash\{0\} \subseteq \mathbb{A}_{k}^{1}$. Therefore, we conclude that there exist an open subscheme $U \subseteq X$ of $X$ and a closed immersion $U \hookrightarrow \mathbb{G}_{m, k} \times_{k} \cdots \times_{k} \mathbb{G}_{m, k}$ over $k$ such that the $\widetilde{\mathbb{A}}_{k}^{f}$-valued point $x_{\mathbb{A}} \stackrel{\text { def }}{=}$ $\left(x_{\mathfrak{p}}\right)_{\mathfrak{p} \in \mathfrak{P}_{k}^{f}}$ of $X$ determined by the fixed $k_{\mathfrak{p}}$-valued points $x_{\mathfrak{p}}$ lies on $U$. On the other hand, again by condition (4), one verifies easily that, for each $i \in\{1, \cdots, N\}$, the $\widetilde{\mathbb{A}}_{k}^{f}$-valued point of $\mathbb{G}_{m, k}$ obtained as the composite

$$
\operatorname{Spec} \widetilde{\mathbb{A}}_{k}^{f} \stackrel{x_{\mathbb{A}}}{\longrightarrow} U \hookrightarrow \mathbb{G}_{m, k} \times_{k} \cdots \times_{k} \mathbb{G}_{m, k} \stackrel{\mathrm{pr}_{i}}{\rightarrow} \mathbb{G}_{m, k}
$$

determines an $\mathbb{A}_{k}^{f}$-valued point of $\mathbb{G}_{m, k}$. Thus, it follows immediately from Lemma 3.4, (ii), (iii); condition (c), that, for each $i \in\{1, \cdots, N\}$, the $k_{\mathfrak{p}_{0}}$-valued point of $\mathbb{G}_{m, k}$ obtained as the composite

$$
\text { Spec } k_{\mathfrak{p}_{0}} \stackrel{x_{\mathfrak{p}_{0}}}{\rightarrow} U \hookrightarrow \mathbb{G}_{m, k} \times_{k} \cdots \times_{k} \mathbb{G}_{m, k} \stackrel{\mathrm{pr}_{i}}{\rightarrow} \mathbb{G}_{m, k}
$$

determines a $k\left(\zeta_{r\left(\mathfrak{p}_{0}\right)}\right)$-valued point of $\mathbb{G}_{m, k}$. In particular, since $U \hookrightarrow$ $\mathbb{G}_{m, k} \times_{k} \cdots \times_{k} \mathbb{G}_{m, k}$ is a closed immersion, one verifies easily that the $k_{\mathfrak{p}_{0}}$-valued point $x_{\mathfrak{p}_{0}}$ of $U$, hence also $X$, determines a $k\left(\zeta_{r\left(\mathfrak{p}_{0}\right)}\right)$-valued point. This completes the proof of the implication $(4) \Rightarrow(1)$, hence also of Theorem 4.5. 
Remark 4.5.1.

(i) Theorem 4.5 is a result without any assumption on the finiteness of a Shafarevich-Tate group.

(ii) The equivalence $(1) \Leftrightarrow(3)$ of Theorem 4.5 may be regarded as a tripod analogue of [4], Theorem 17. The condition that $k$ is either the field of rational numbers or an imaginary quadratic field [i.e., the assumption that $\mathfrak{o}_{k}^{\times}$is finite] in the statement of Theorem 4.5 may be regarded as an analogue of the finiteness condition on the Mordell-Weil group in the statement of [4], Theorem 17; the condition that $\operatorname{Pic}\left(\mathfrak{o}_{k}\right)$ is annihilated by a $\Sigma(\mathcal{C})$-integer in the statement of Theorem 4.5 may be regarded as an analogue of the finiteness condition on the Shafarevich-Tate group in the statement of [4], Theorem 17. On the other hand, since any abelian variety is proper, in the case of [4], Theorem 17, the condition corresponding to our condition that the birational Galois section determines [not only an $\widetilde{\mathbb{A}}_{k}^{f}$-valued point but also] an $\mathbb{A}_{k}^{f}$-valued point of the tripod $\mathbb{P}_{k}^{1} \backslash\{0,1, \infty\}$ in Theorem 4.5 is automatically satisfied.

(iii) If $\mathcal{C}$ contains all finite solvable groups, then Theorem $4.1 \mathrm{im}-$ plies the equivalence $(1) \Leftrightarrow(4)$ of Theorem 4.5 .

(iv) One verifies easily that the equivalence $(1) \Leftrightarrow(4)$ of Theorem 4.5 gives us an alternative proof of Corollary 4.2 in the case where $s$ is a pro-C birational Galois section, and $k$ is either the field of rational numbers or an imaginary quadratic field.

Corollary 4.6. Let $k$ be either the field of rational numbers or an imaginary quadratic field and $\bar{k}$ an algebraic closure of $k$. Write $G_{k} \stackrel{\text { def }}{=}$ $\operatorname{Gal}(\bar{k} / k)$ and $\mathfrak{P}_{k}^{f}$ for the set of nonarchimedean primes of $k$. For each $\mathfrak{p} \in \mathfrak{P}_{k}^{f}$, write $k_{\mathfrak{p}}$ for the $\mathfrak{p}$-adic completion of $k$. Then the following assertions are equivalent:

(1) Any pro-Primes birational Galois section [cf. Definition 1.2] of any projective smooth curve over $k$ [where we refer to the discussion entitled "Curves" in $\S 0$ concerning the term "smooth curve"] is geometric [cf. Definition 1.3].

(2) Any pro-Primes birational Galois section of $\mathbb{P}_{k}^{1} / k$ is geometric.

(3) Any pro-Primes birational Galois section $s$ of $\mathbb{P}_{k}^{1} / k$ satisfies the following two conditions:

(3-i) There exist three distinct elements $a, b, c \in \mathbb{P}_{k}^{1}(k)$ of $\mathbb{P}_{k}^{1}(k)$ such that, for any nonarchimedean prime $\mathfrak{p}$ of $k$, the [uniquely determined - cf. Lemma 2.7] $k_{\mathfrak{p}}$-valued point of $\mathbb{P}_{k}^{1}$ associated to $s$ [cf. Definition 2.6; Proposition 2.9] is $\notin\{a, b, c\} \subseteq$ $\left(\mathbb{P}_{k}^{1}(k) \subseteq\right) \mathbb{P}_{k}^{1}\left(k_{\mathfrak{p}}\right)$.

(3-ii) There exists a prime number $l$ such that the pro-l Galois section of $\mathbb{P}_{k}^{1} \backslash\{0,1, \infty\}$ [cf. Definition 1.2] naturally determined by 
$s$ is either cuspidal [cf. Definition 4.3, (i)] or unramified almost everywhere $[c f$. Definition 4.3 , (ii)].

(4) Any pro-Primes birational Galois section $s$ of $\mathbb{P}_{k}^{1} / k$ satisfies the following two conditions:

(4-i) There exist three distinct elements $a, b, c \in \mathbb{P}_{k}^{1}(k)$ of $\mathbb{P}_{k}^{1}(k)$ such that, for any nonarchimedean prime $\mathfrak{p}$ of $k$, the [uniquely determined - cf. Lemma 2.7] $k_{\mathfrak{p}}$-valued point of $\mathbb{P}_{k}^{1}$ associated to $s$ [cf. Definition 2.6; Proposition 2.9] is $\notin\{a, b, c\} \subseteq$ $\left(\mathbb{P}_{k}^{1}(k) \subseteq\right) \mathbb{P}_{k}^{1}\left(k_{\mathfrak{p}}\right)$.

(4-ii) Write $s^{\mathbb{P}}$ for the pro-Primes Galois section of $\mathbb{P}_{k}^{1} \backslash\{0,1, \infty\}$ naturally determined by $s$. Then it holds either that $s^{\mathbb{P}}$ is cuspidal, or that there exists a prime number l such that the l-adic Galois representation

$$
G_{k} \stackrel{s^{\mathbb{P}}}{\longrightarrow} \Pi_{\left(\mathbb{P}_{k}^{1} \backslash\{0,1, \infty\}\right) / k}^{\mathfrak{P r i m e s}} \longrightarrow \mathrm{GL}_{2}\left(\mathbb{Z}_{l}\right)
$$

- where we refer to Definition 1.1, (ii), concerning the profinite group $\Pi_{\left(\mathbb{P}_{k}^{1} \backslash\{0,1, \infty\}\right) / k^{2}}^{\mathfrak{P r i m e s}} ;$ the second arrow $\prod_{\left(\mathbb{P}_{k}^{1} \backslash\{0,1, \infty\}\right) / k}^{\mathfrak{P r i m e \mathfrak { s }}} \rightarrow$ $\mathrm{GL}_{2}\left(\mathbb{Z}_{l}\right)$ is the l-adic representation determined by the Legendre family of elliptic curves over $\mathbb{P}_{k}^{1} \backslash\{0,1, \infty\}$, i.e., the elliptic curve over $\mathbb{P}_{k}^{1} \backslash\{0,1, \infty\}=\operatorname{Spec} k\left[u^{ \pm 1},(1-u)^{-1}\right]$ determined by the equation " $y^{2}=x(x-1)(x-u)$ " - is unramified at all but finitely many $\mathfrak{p} \in \mathfrak{P}_{k}^{f}$.

Proof. The implications $(1) \Rightarrow(2) \Rightarrow(4)$ are immediate [cf. also Remark 2.6.1]. On the other hand, the implication $(2) \Rightarrow(1)$ follows immediately from the fact that any projective smooth curve over $k$ may be obtained as the normalization of $\mathbb{P}_{k}^{1}$ in the finite extension of $k\left(\mathbb{P}_{k}^{1}\right)$ corresponding to an open subgroup of $\Pi_{k\left(\mathbb{P}_{k}^{1}\right) / k}^{\mathfrak{P r i m e s}}$. Moreover, let us observe that it follows immediately from Proposition 4.4, together with Remark 2.6.1, that the implications $(2) \Rightarrow(3)$ holds.

Finally, we verify the implication $(3) \Rightarrow(2)$ (respectively, $(4) \Rightarrow$ (2)). Suppose that assertion (3) (respectively, assertion (4)) holds. Let $s$ be a pro-Primes birational Galois section of $\mathbb{P}_{k}^{1} / k$. For each nonarchimedean prime $\mathfrak{p}$ of $k$, write $x_{\mathfrak{p}}$ for the [uniquely determined] $k_{\mathfrak{p}^{-}}$ valued point of $\mathbb{P}_{k}^{1}$ associated to $s$. Then it follows from condition (3-i) (respectively, condition (4-i)) that, by considering a suitable automorphism of $\mathbb{P}_{k}^{1}$ over $k$, we may assume without loss of generality that, for any $\mathfrak{p} \in \mathfrak{P}_{k}^{f}, x_{\mathfrak{p}} \in \mathbb{P}_{k}^{1}\left(k_{\mathfrak{p}}\right)$ is $\notin\{0,1, \infty\} \subseteq \mathbb{P}_{k}^{1}\left(k_{\mathfrak{p}}\right)$. Thus, for any prime number $l$, by applying Lemma 1.4 [to " $x_{\mathfrak{p}}$ " for $\mathfrak{p} \in \mathfrak{P}_{k}^{f}$ whose residue characteristic is $=l]$, it follows immediately that the pro-l Galois section $s^{\mathbb{P},\{l\}}$ of $\mathbb{P}_{k}^{1} \backslash\{0,1, \infty\}$ obtained as the composite

$$
G_{k} \stackrel{s}{\longrightarrow} \prod_{k\left(\mathbb{P}_{k}^{1}\right) / k}^{\mathfrak{P r i m e s}} \longrightarrow \prod_{\left(\mathbb{P}_{k}^{1} \backslash\{0,1, \infty\}\right) / k}^{\{l\}}
$$


hence also the pro-Primes Galois section $s^{\mathbb{P}}$ of $\mathbb{P}_{k}^{1} \backslash\{0,1, \infty\}$ obtained as the composite

$$
G_{k} \stackrel{s}{\longrightarrow} \prod_{k\left(\mathbb{P}_{k}^{1}\right) / k}^{\mathfrak{P r i m e s}} \longrightarrow \prod_{\left(\mathbb{P}_{k}^{1} \backslash\{0,1, \infty\}\right) / k}^{\mathfrak{P r i m e s}}
$$

is not cuspidal. Thus, by condition (3-ii) (respectively, condition (4ii)), we conclude that there exists a prime number $l_{0}$ such that $s^{\mathbb{P}},\left\{l_{0}\right\}$ is unramified almost everywhere (respectively, the $l_{0}$-adic Galois representation obtained as the displayed composite of condition (4-ii) is unramified at all but finitely many $\mathfrak{p} \in \mathfrak{P}_{k}^{f}$ ). Thus, since [we have assumed that] for any $\mathfrak{p} \in \mathfrak{P}_{k}^{f}, x_{\mathfrak{p}} \in \mathbb{P}_{k}^{1}\left(k_{\mathfrak{p}}\right)$ is $\notin\{0,1, \infty\} \subseteq \mathbb{P}_{k}^{1}\left(k_{\mathfrak{p}}\right)$, it follows immediately from Proposition A.7 (respectively, [13], Theorem 1) that the birational pro-Primes Galois section $s$ of $\mathbb{P}_{k}^{1} / k$ satisfies condition (3) of Theorem 4.5, hence also [by the equivalence $(1) \Leftrightarrow(3)$ of Theorem 4.5] that $s$ is geometric. This completes the proof of the implication $(3) \Rightarrow(2)$ (respectively, $(4) \Rightarrow(2)$ ), hence also of Corollary 4.6.

\section{APPENDIX A. RAMIFICATION OF GALOIS SECTIONS}

In the present $\S \mathrm{A}$, we discuss the ramification of Galois sections of hyperbolic curves over $p$-adic local fields. In the present $\S \mathrm{A}$, let $\Sigma \subseteq$ Primes be a nonempty subset of Primes [where we refer to the discussion entitled "Numbers" in $\S 0$ concerning the set $\mathfrak{P r i m e s}], k$ a $p$-adic local field for some prime number $p$ [where we refer to the discussion entitled "Numbers" in $\S 0$ concerning the term " $p$-adic local field"], $\bar{k}$ an algebraic closure of $k$, and $X$ a hyperbolic curve over $k$ [where we refer to the discussion entitled "Curves" in $\S 0$ concerning the term "hyperbolic curve"]. For a finite extension $k^{\prime}(\subseteq \bar{k})$ of $k$, write

$$
\begin{gathered}
G_{k^{\prime}} \stackrel{\text { def }}{=} \operatorname{Gal}\left(\bar{k} / k^{\prime}\right), \\
I_{k^{\prime}} \subseteq G_{k^{\prime}}
\end{gathered}
$$

for the inertia subgroup of $G_{k^{\prime}}$, and

$$
\mathfrak{o}_{k^{\prime}} \subseteq k^{\prime}
$$

for the ring of integers of $k^{\prime}$. Write, moreover,

$$
X^{\mathrm{cpt}}
$$

for the [uniquely determined] smooth compactification of $X$ over $k$;

$$
\Delta_{X / k}^{\Sigma}
$$

for the pro- $\Sigma$ geometric fundamental group of $X$, i.e., the maximal pro- $\Sigma$ quotient of $\pi_{1}\left(X \otimes_{k} \bar{k}\right)$;

$$
\Pi_{X / k}^{\Sigma}
$$

for the geometrically pro- $\Sigma$ fundamental group of $X$, i.e., the quotient of $\pi_{1}(X)$ by the kernel of the natural surjection $\pi_{1}\left(X \otimes_{k} \bar{k}\right) \rightarrow \Delta_{X / k}^{\Sigma}$. 
Thus, we have an exact sequence of profinite groups [cf. [3], Exposé IX, Théorème 6.1]

$$
1 \longrightarrow \Delta_{X / k}^{\Sigma} \longrightarrow \Pi_{X / k}^{\Sigma} \longrightarrow G_{k} \longrightarrow 1 \text {. }
$$

Let $s$ be a pro- $\Sigma$ Galois section of $X / k$ [cf. [6], Definition 1.1, (i)], i.e., a section of the above exact sequence of profinite groups.

Definition A.1. We shall say that $s$ is unramified (respectively, potentially unramified) if the image of the composite

$$
I_{k} \longrightarrow G_{k} \stackrel{s}{\longrightarrow} \Pi_{X / k}^{\Sigma} \longrightarrow \operatorname{Aut}\left(\Delta_{X / k}^{\Sigma}\right)
$$

- where the third arrow is the action of $\Pi_{X / k}^{\Sigma}$ on $\Delta_{X / k}^{\Sigma}$ obtained by conjugation - is trivial (respectively, finite).

Proposition A.2. The following hold:

(i) If $p \in \Sigma$, then any pro- $\Sigma$ Galois section of $X / k$ [cf. [6], Definition 1.1, (i)] is not potentially unramified, hence also not unramified [cf. Definition A.1].

(ii) If $X$ does not admit good reduction over $\mathfrak{o}_{k}$ [where we refer to the discussion entitled "Curves" in $§ 0$ concerning the term "good reduction"], then any pro- $\Sigma$ Galois section of $X / k$ is not unramified.

Proof. Let $s$ be a pro- $\Sigma$ Galois section of $X / k$. Now one verifies easily that there exists a characteristic open subgroup $H \subseteq \Delta_{X / k}^{\Sigma}$ of $\Delta_{X / k}^{\Sigma}$ such that the connected finite étale covering of $X$ corresponding to the open subgroup $H \cdot \operatorname{Im}(s)$ of $\Pi_{X / k}^{\Sigma}$ topologically generated by $H$ and $\operatorname{Im}(s)$ is of genus $\geq 1$. On the other hand, since $H \subseteq \Delta_{X / k}^{\Sigma}$ is characteristic, and $\Delta_{X / k}^{\Sigma}$ is slim [where we refer to the discussion entitled "Profinite groups" in $\S 0$ concerning the term "slim"], it follows from [7], Lemma 5, that we have a natural injection $\operatorname{Aut}\left(\Delta_{X / k}^{\Sigma}\right) \hookrightarrow \operatorname{Aut}(H)$. Thus, to verify Proposition A.2, by replacing $\Pi_{X / k}^{\Sigma}$ by the open subgroup $H \cdot \operatorname{Im}(s)$, we may assume without loss of generality that $X$ is of genus $\geq 1$.

Now we verify assertion (i). As is well-known, if $p \in \Sigma$, then there exist $G_{k}$-equivariant isomorphisms

$$
H^{2}\left(\Delta_{X^{\mathrm{cpt}} / k}^{\Sigma}, \mathbb{Z}_{p}\right) \simeq H^{2}\left(X^{\mathrm{cpt}} \otimes_{k} \bar{k}, \mathbb{Z}_{p}\right) \simeq \mathbb{Z}_{p}(1)
$$

— where " $(1)$ " denotes a Tate twist. In particular, [the restriction to $I_{k}$ of] the $p$-adic cyclotomic representation $\chi_{p}: I_{k} \rightarrow \operatorname{Aut}\left(\mathbb{Z}_{p}(1)\right)$ factors through the displayed composite of Definition A.1. On the other hand, one may verify easily that the image of $\chi_{p}$ is infinite. Thus, $s$ is not potentially unramified. This completes the proof of assertion (i).

Next, we verify assertion (ii). Suppose that $X$ does not admit good reduction over $\mathfrak{o}_{k}$. Now it follows from assertion (i) that, to verify assertion (ii), by replacing $\Sigma$ by $\Sigma \backslash(\Sigma \cap\{p\})$, we may assume without 
loss of generality that $p \notin \Sigma$. Then it follows immediately from [15], Theorem 0.8 , that the image of the composite

$$
I_{k} \longrightarrow \operatorname{Aut}\left(\Delta_{X / k}^{\Sigma}\right) \longrightarrow \operatorname{Out}\left(\Delta_{X / k}^{\Sigma}\right)
$$

- where the first arrow is the displayed composite of Definition A.1 - is nontrivial, hence that $s$ is not unramified. This completes the proof of assertion (ii).

Definition A.3. If $X$ admits good reduction $\mathcal{X}$ over $\mathfrak{o}_{k}$, then we shall write

$$
\left(\pi_{1}(X) \rightarrow \Pi_{X / k}^{\Sigma} \rightarrow\right) \Pi_{X / k}^{\Sigma \text {-ét }}
$$

for the quotient of $\pi_{1}(X)$ by the normal closed subgroup topologically normally generated by the kernels of the natural surjections $\pi_{1}(X) \rightarrow \Pi_{X / k}^{\Sigma}, \pi_{1}(X) \rightarrow \pi_{1}(\mathcal{X})$. Thus, the natural surjection $\Pi_{X / k}^{\Sigma} \rightarrow$ $G_{k}$ determines a surjection $\Pi_{X / k}^{\Sigma \text {-ét }} \rightarrow G_{k} / I_{k}$. We shall write

$$
\Delta_{X / k}^{\Sigma-e ́ t}
$$

for the kernel of the surjection $\Pi_{X / k}^{\Sigma \text {-ét }} \rightarrow G_{k} / I_{k}$. Thus, we have a commutative diagram of profinite groups

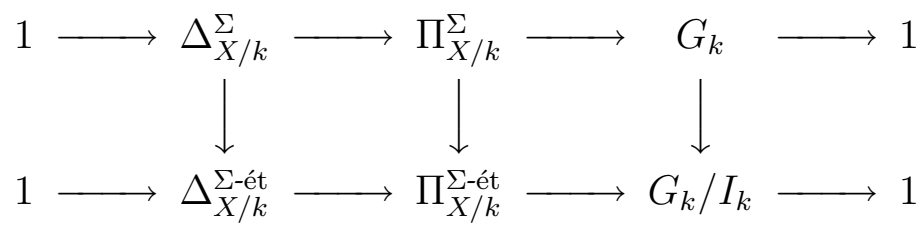

- where the horizontal sequences are exact.

Remark A.3.1. In the notation of Definition A.3, as is well-known, if $p \notin \Sigma$, then the left-hand vertical arrow $\Delta_{X / k}^{\Sigma} \rightarrow \Delta_{X / k}^{\Sigma \text {-ét }}$ of the commutative diagram of Definition A.3 is an isomorphism; in particular, the right-hand upper horizontal arrow $\Pi_{X / k}^{\Sigma} \rightarrow G_{k}$ induces an isomorphism $\operatorname{Ker}\left(\Pi_{X / k}^{\Sigma} \rightarrow \Pi_{X / k}^{\Sigma \text {-ét }}\right) \stackrel{\sim}{\rightarrow} I_{k}$.

Proposition A.4. The following conditions are equivalent:

(1) $s$ is unramified [cf. Definition A.1].

(2) $p \notin \Sigma, X$ admits good reduction over $\mathfrak{o}_{k}$, and the image of the composite

$$
I_{k} \hookrightarrow G_{k} \stackrel{s}{\rightarrow} \Pi_{X / k}^{\Sigma} \rightarrow \Pi_{X / k}^{\Sigma \text {-ét }}
$$

[cf. Definition A.3] is trivial.

(3) $p \notin \Sigma, X$ admits good reduction over $\mathfrak{o}_{k}$, and the composite

$$
I_{k} \hookrightarrow G_{k} \stackrel{s}{\rightarrow} \Pi_{X / k}^{\Sigma}
$$

determines an isomorphism

$$
I_{k} \stackrel{\sim}{\longrightarrow} \operatorname{Ker}\left(\Pi_{X / k}^{\Sigma} \rightarrow \Pi_{X / k}^{\Sigma-e ́ t}\right) .
$$


(4) $p \notin \Sigma$, and, for any open subgroup $H \subseteq \Pi_{X / k}^{\Sigma}$ of $\Pi_{X / k}^{\Sigma}$ containing the image of $s$, the connected finite étale covering of $X$ corresponding to $H \subseteq \Pi_{X / k}^{\Sigma}$ admits good reduction over $\mathfrak{o}_{k}$.

Proof. First, we verify the equivalence $(1) \Leftrightarrow(2)$. It follows immediately from Proposition A.2 that both (1) and (2) imply that $p \notin \Sigma$, and that $X$ admits good reduction over $\mathfrak{o}_{k}$. Thus, suppose that these conditions are satisfied. Write $J$ for the image of the displayed composite of condition (2). Then it follows immediately from the existence of the commutative diagram of Definition A.3 that $J \subseteq \Delta_{X / k}^{\Sigma \text {-ét }} \subseteq \Pi_{X / k}^{\Sigma \text {-ét }}$. Thus, it follows immediately from Remark A.3.1 that the displayed composite $I_{k} \rightarrow \operatorname{Aut}\left(\Delta_{X / k}^{\Sigma}\right)$ of Definition A.1 factors as

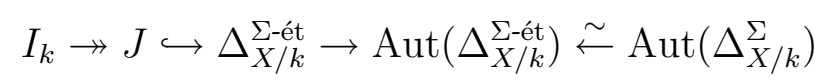

- where the third arrow is the action of $\Delta_{X / k}^{\Sigma \text {-ét }}$ on $\Delta_{X / k}^{\Sigma \text {-ét }}$ obtained by conjugation. Now since, as is well-known, $\Delta_{X / k}^{\Sigma} \stackrel{\sim}{\rightarrow} \Delta_{X / k}^{\sum \text {-ét is center- }}$ free, the third arrow of this composite is injective. Therefore, it follows immediately that the condition that $s$ is unramified is equivalent to the condition that $J=\{1\}$. This completes the proof of the equivalence $(1) \Leftrightarrow(2)$.

The equivalence $(2) \Leftrightarrow(3)$ follows immediately from Remark A.3.1. Next, we verify the implication $(3) \Rightarrow(4)$. Suppose that condition (3) is satisfied. Then it is immediate that if an open subgroup of $\Pi_{X / k}^{\Sigma}$ contains the image of $s$, then it arises from an open subgroup of $\Pi_{X / k}^{\Sigma \text {-ét. }}$; thus, it follows immediately from the various definitions involved that the corresponding connected finite étale covering of $X$ admits good reduction over $\mathfrak{o}_{k}$. This completes the proof of the implication $(3) \Rightarrow(4)$.

Finally, we verify the implication $(4) \Rightarrow(3)$. Suppose that condition (4) is satisfied. Let $H \subseteq \Pi_{X / k}^{\Sigma}$ be an open subgroup of $\Pi_{X / k}^{\Sigma}$ containing the image of $s$. Write $Y \rightarrow X$ for the connected finite étale covering of $X$ corresponding to $H \subseteq \Pi_{X / k}^{\Sigma}$; thus, $\Pi_{Y / k}^{\Sigma}=H \subseteq \Pi_{X / k}^{\Sigma}$. Then it follows from condition (4) that $Y$ admits good reduction over $\mathfrak{o}_{k}$. Thus, since $p \notin \Sigma$, it follows from [15], Lemma 5.5, that the connected finite étale covering $Y \rightarrow X$ extends to a connected finite étale covering of the [uniquely determined] smooth model of $X$ over $\mathfrak{o}_{k}$. In particular, it follows immediately from the various definitions involved that $H \subseteq \Pi_{X / k}^{\Sigma}$ arises from an open subgroup of $\Pi_{X / k}^{\Sigma \text {-ét }}$ i.e., $\operatorname{Ker}\left(\Pi_{X / k}^{\Sigma} \rightarrow \Pi_{X / k}^{\Sigma \text {-ét }}\right) \subseteq H$. Therefore, by considering the intersection of such $H^{\prime}$ s, we obtain that $\operatorname{Ker}\left(\Pi_{X / k}^{\Sigma} \rightarrow \Pi_{X / k}^{\Sigma \text {-ét }}\right) \subseteq \operatorname{Im}(s)$. Thus, by Remark A.3.1, we conclude that condition (3) holds. This completes the proof of the implication $(4) \Rightarrow(3)$, hence also of Proposition A.4. 
Lemma A.5. Suppose that $p \notin \Sigma$, and that $X$ admits good reduction over $\mathfrak{o}_{k}$. Let $\Pi \subseteq \Pi_{X / k}^{\Sigma \text {-ét }}$ be an open subgroup of $\Pi_{X / k}^{\Sigma \text {-ét. }}$. Write $s_{\mathfrak{o}}$ for the composite $G_{k} \stackrel{s}{\rightarrow} \Pi_{X / k} \rightarrow \Pi_{X / k^{\prime}}^{\Sigma \text {-é }} k^{\prime}(\subseteq \bar{k})$ for the [necessarily unramified] finite extension of $k$ corresponding to the image of the composite $\Pi \hookrightarrow \Pi_{X / k}^{\Sigma \text {-ét }} \rightarrow$ $G_{k} / I_{k}, Y \rightarrow X$ for the connected finite étale covering of $X$ corresponding to the open subgroup $\Pi \subseteq \Pi_{X / k^{\prime}}^{\Sigma \text {-ét }}$ and $Y^{\mathrm{cpt}}$ for the [uniquely determined] smooth compactification of $Y$ over $k^{\prime}$. [Here, it follows immediately from the various definitions involved that $Y$ is a hyperbolic curve over $k^{\prime} ; Y$, hence also $Y^{\mathrm{cpt}}$, admits good reduction over $\mathfrak{o}_{k^{\prime}} ; \Pi_{Y / k^{\prime}}^{\Sigma \text {-ét }}=\Pi \subseteq \Pi_{X / k^{\circ}}^{\Sigma \text {-ét }}$. Suppose, moreover, that $Y$ is of genus $\geq 2$. Then the image of the composite

$$
s_{\mathfrak{o}}\left(I_{k}\right) \cap \Delta_{Y / k^{\prime}}^{\sum \text {-ét }} \hookrightarrow \Delta_{Y / k^{\prime}}^{\sum \text {-ét }} \rightarrow \Delta_{Y^{\mathrm{cpt}} / k^{\prime}}^{\sum \text {-ét }} \rightarrow\left(\Delta_{Y^{\mathrm{cpt}} / k^{\prime}}^{\sum-\mathrm{e}^{\prime}}\right)^{\mathrm{ab}}
$$

- where the second arrow is the surjection induced by the open immersion $Y \hookrightarrow Y^{\mathrm{cpt}}$, and we refer to the discussion entitled "Profinite groups" in $\S 0$ concerning $\left(\Delta_{Y^{\mathrm{cpt}} / k^{\prime}}^{\sum-e^{\prime}}\right)^{\mathrm{ab}}-i$ trivial.

Proof. Write $\mathcal{Y}^{\mathrm{cpt}}$ for the smooth model of $Y^{\mathrm{cpt}}$ over $\mathfrak{o}_{k^{\prime}}$. Then, to verify Lemma A.5, by replacing $k^{\prime}$ by a finite unramified extension of $k^{\prime}$, we may assume without loss of generality that $\mathcal{Y}^{\operatorname{cpt}}\left(\mathfrak{o}_{k^{\prime}}\right) \neq \emptyset$. Moreover, since, as is well-known [cf. also Remark A.3.1], $\left(\Delta_{Y^{\mathrm{cpt}} / k^{\prime}}^{\sum \text {-ét }}\right)^{\mathrm{ab}}$ is a free $\widehat{\mathbb{Z}}^{\Sigma}$-module, in light of the existence of the commutative diagram of Definition A.3, to verify Lemma A.5, by replacing $k$ by a finite extension of $k$, we may assume without loss of generality that $s_{\mathfrak{o}}\left(I_{k^{\prime}}\right) \subseteq \Delta_{Y / k^{\prime}}^{\Sigma}$; thus, to verify Lemma A.5, it suffices to verify that the image of the composite

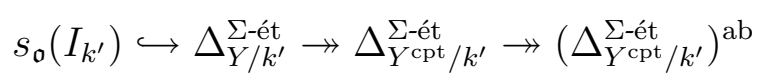

is trivial. Write $J$ for the Jacobian variety of $Y^{\mathrm{cpt}}$ over $k^{\prime}$ and $\mathcal{J}$ for the smooth model of $J$ over $\mathfrak{o}_{k^{\prime}}$. Then, as is well-known, the closed immersion $\mathcal{Y}^{\text {cpt }} \hookrightarrow \mathcal{J}$ over $\mathfrak{o}_{k^{\prime}}$ determined by an $\mathfrak{o}_{k^{\prime}}$-rational point of $\mathcal{Y}^{\mathrm{cpt}}$ induces an isomorphism

$$
\Pi_{Y^{\mathrm{cpt}} / k^{\prime}}^{\sum \text {-ét }} /\left[\Delta_{Y^{\mathrm{cpt}} / k^{\prime}}^{\sum \text {-ét }}, \Delta_{Y^{\mathrm{cpt}} / k^{\prime}}^{\sum \text {-ét }}\right] \stackrel{\sim}{\longrightarrow} \Pi_{J / k^{\prime}}^{\sum \text {-ét }}
$$

- where we write $\Pi_{J / k^{\prime}}^{\Sigma \text {-ét }}$ for the quotient of $\pi_{1}(J)$ by the normal closed subgroup topologically normally generated by the kernels of the natural surjections $\pi_{1}(J) \rightarrow \Pi_{J / k^{\prime}}^{\Sigma}, \pi_{1}(J) \rightarrow \pi_{1}(\mathcal{J})$ - over $G_{k^{\prime}} / I_{k^{\prime}}$. In particular, it follows immediately from the various definitions involved that, to verify Lemma A.5, it suffices to verify that the image of the homomorphism $I_{k^{\prime}} \rightarrow \Delta_{J / k^{\prime}}^{\Sigma \text {-ét }}$ - where we write $\Delta_{J / k^{\prime}}^{\Sigma \text {-ét }}$ for the kernel of the natural surjection $\Pi_{J / k^{\prime}}^{\Sigma \text {-ét }} \rightarrow G_{k^{\prime}} / I_{k^{\prime}}$, which is, as is wellknown, naturally isomorphic to $\Delta_{J / k^{\prime}}^{\Sigma}$ - determined by the composite

$$
I_{k^{\prime}} \stackrel{s_{0}}{\rightarrow} \Pi_{Y / k^{\prime}}^{\sum-e^{\prime}} \rightarrow \Pi_{J / k^{\prime}}^{\sum-e ́ t}
$$


is trivial. On the other hand, one verifies easily that this homomorphism $I_{k^{\prime}} \rightarrow \Delta_{J / k^{\prime}}^{\Sigma \text {-ét }}$ is compatible with the respective natural outer actions of $G_{k^{\prime}} / I_{k^{\prime}}$; moreover, since $\Delta_{J / k^{\prime}}^{\Sigma \text {-ét }}$ is pro- $\Sigma$, this homomorphism

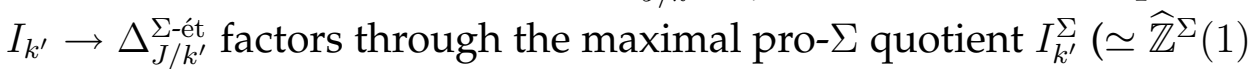
- where "(1)" denotes a Tate twist) of $I_{k^{\prime}}$. Therefore, by comparing the weights of the actions of the Frobenius element in $G_{k^{\prime}} / I_{k^{\prime}}$ on $I_{k^{\prime}}^{\Sigma}\left(\simeq \widehat{\mathbb{Z}}^{\Sigma}(1)\right)$ [which is of weight 2] and $\Delta_{J / k^{\prime}}^{\Sigma \text {-ét }}$ [which is of weight 1 — cf., e.g., [11], §21, Theorem 4], it follows immediately from the well-known fact that $\Delta_{J / k^{\prime}}^{\Sigma \text {-ét }}$ is a free $\widehat{\mathbb{Z}}^{\Sigma}$-module that the image of the homomorphism $I_{k^{\prime}}^{\Sigma} \rightarrow \Delta_{J / k^{\prime}}^{\Sigma \text {-ét }}$ is trivial. This completes the proof of Lemma A.5.

Proposition A.6. Suppose that $p \notin \Sigma$, and that $X$ admits good reduction over $\mathfrak{o}_{k}$. Then the following conditions are equivalent:

(1) $s$ is ramified, i.e., not unramified [cf. Definition A.1].

(2) The image of the composite

$$
I_{k} \hookrightarrow G_{k} \stackrel{s}{\rightarrow} \Pi_{X / k}^{\Sigma} \rightarrow \Pi_{X / k}^{\Sigma-e ́ t}
$$

is a nontrivial closed subgroup of a cuspidal inertia subgroup of $\Pi_{X / k}^{\Sigma-\text {-ét }}$ associated to a cusp of $X / k$.

(3) The image of the composite

$$
I_{k} \hookrightarrow G_{k} \stackrel{s}{\rightarrow} \Pi_{X / k}^{\Sigma} \rightarrow \Phi_{X / k}^{\Sigma}
$$

- where $\Phi_{X / k}^{\Sigma}$ is the quotient of $\Pi_{X / k}^{\Sigma}$ defined in [7], Definition 1 , (iv), i.e., the quotient of $\Pi_{X / k}^{\Sigma}$ by the kernel $Z_{\Pi_{X / k}^{\Sigma}}\left(\Delta_{X / k}^{\Sigma}\right)$ of the homomorphism $\Pi_{X / k}^{\Sigma} \rightarrow \operatorname{Aut}\left(\Delta_{X / k}^{\Sigma}\right)$ obtained by conjugation - is a nontrivial closed subgroup of a cuspidal inertia subgroup of $\Phi_{X / k}^{\Sigma}$ associated to a cusp of $X / k$.

(4) There exists an element $l \in \Sigma$ of $\Sigma$ such that the pro-l Galois section of $X / k[c f$. [6], Definition 1.1, (i)] naturally determined by $s$ is ramified.

Proof. First, we verify the equivalence $(1) \Leftrightarrow(2)$. It follows from the equivalence $(1) \Leftrightarrow(2)$ of Lemma A.4 that $s$ is ramified if and only if the image of the composite of condition (2) is nontrivial. On the other hand, it follows immediately from the existence of the commutative diagram of Definition A.3, together with Remark A.3.1, that the composite of condition (2) factors through the maximal pro$\Sigma$ quotient of $I_{k}$, which is, as is well-known, procyclic. Thus, it follows immediately from Lemma A.5, together with [5], Lemma 1.6, that the equivalence $(1) \Leftrightarrow(2)$ holds. This completes the proof of the equivalence $(1) \Leftrightarrow(2)$. Next, let us observe that the implication $(3) \Rightarrow(1)$ follows immediately from the various definitions involved [cf. also the definition of the quotient $\Phi_{X / k}^{\Sigma}$ ]. Next, we 
verify the implication $(2) \Rightarrow(3)$. Since, as is well-known, $\Delta_{X / k}^{\Sigma}$ is slim [where we refer to the discussion entitled "Profinite groups" in $\S 0$ concerning the term "slim"], it follows from [7], Proposition 6, (ii), together with Remark A.3.1, that we have a sequence of natural surjections $\Pi_{X / k}^{\Sigma} \rightarrow \Pi_{X / k}^{\Sigma \text {-ét }} \rightarrow \Phi_{X / k}^{\Sigma}$, which induces an injection $\Delta_{X / k}^{\Sigma} \stackrel{\sim}{\rightarrow} \Delta_{X / k}^{\Sigma-e ́ t ~} \hookrightarrow \Phi_{X / k}^{\Sigma}$. Thus, one verifies easily that the implication $(2) \Rightarrow(3)$ holds. This completes the proof of the implication $(2) \Rightarrow(3)$. Finally, we verify the equivalence $(1) \Leftrightarrow(4)$. For each nonempty subset $\Sigma^{\prime} \subseteq \Sigma$ of $\Sigma$, write $J_{\Sigma^{\prime}}$ for the image of the composite

$$
I_{k} \hookrightarrow G_{k} \stackrel{s}{\rightarrow} \Pi_{X / k}^{\Sigma} \rightarrow \Pi_{X / k}^{\Sigma^{\prime}} \rightarrow \Pi_{X / k}^{\Sigma^{\prime}-\text { ét }} .
$$

Then it follows immediately from the verified equivalence $(1) \Leftrightarrow(2)$, together with well-known structure of the maximal pro- $\Sigma$ quotient of the fundamental group of a smooth curve over an algebraically closed field of characteristic $\notin \Sigma$, that, for each nonempty subset $\Sigma^{\prime} \subseteq$ $\Sigma$ of $\Sigma$, the image $J_{\Sigma^{\prime}}$ is procyclic, and, moreover, $J_{\Sigma^{\prime}}$ is the maximal pro- $\Sigma^{\prime}$ quotient of $J_{\Sigma}$ [relative to the natural surjection $J_{\Sigma} \rightarrow J_{\Sigma^{\prime}}$ ]. In particular, we conclude that $J_{\Sigma}=\{1\}$ if and only if $J_{\{l\}}=\{1\}$ for any $l \in \Sigma$, i.e., the equivalence $(1) \Leftrightarrow(4)$ holds. This completes the proof of the equivalence $(1) \Leftrightarrow(4)$, hence also of Proposition A.6.

Proposition A.7. Suppose that $p \notin \Sigma$, and that $s$ is geometric [cf. [6], Definition 1.1, (iii)]. Let $x \in X^{\mathrm{cpt}}(k)$ be a $k$-rational point of $X^{\mathrm{cpt}}$ such that a decomposition subgroup of $\Pi_{X / k}^{\Sigma}$ associated to $x$ contains the image of $s$. Consider the following three conditions:

(1) $s$ is unramified [cf. Definition A.1].

(2) $x \in X(k)$, and the hyperbolic curve $X \backslash\{x\}$, hence also the hyperbolic curve $X$, over $k$ admits good reduction over $\mathfrak{o}_{k}$.

(3) $x \notin X(k)$.

Then we have implications

$$
(2) \Longrightarrow(1) \Longrightarrow \text { either }(2) \text { or }(3) \text {. }
$$

In particular, if $x \in X(k)$, then we have an equivalence

$$
(1) \Longleftrightarrow(2) \text {. }
$$

Proof. To verify Proposition A.7, it is immediate that it suffices to verify that if $x \in X(k)$, then condition (1) is equivalent to condition (2). Thus, suppose that $x \in X(k)$. Now let us observe that it follows immediately from Proposition A.2, (ii), that both (1) and (2) imply that $X$ admits good reduction over $\mathfrak{o}_{k}$. Thus, we may assume without loss of generality that $X$ admits good reduction over $\mathfrak{o}_{k}$. Moreover, observe that it follows immediately from the equivalence $(1) \Leftrightarrow(4)$ of Proposition A.6 that, to verify the equivalence $(1) \Leftrightarrow(2)$, by considering the pro- $l$ Galois section of $X / k$ naturally determined by $s-$ where $l$ ranges over elements of $\Sigma$ - we may assume without loss of 
generality that $\Sigma$ is of cardinality 1 . On the other hand, since $\Sigma$ is of cardinality 1, it follows immediately from [7], Proposition 19, (ii), that the kernel of the composite $G_{k} \stackrel{s}{\rightarrow} \Pi_{X / k}^{\Sigma} \rightarrow \operatorname{Aut}\left(\Delta_{X / k}^{\Sigma}\right)$ coincides with the kernel of the pro- $\Sigma$ outer Galois representation associated to the hyperbolic curve $X \backslash\{x\}$ over $k$. Thus, the equivalence $(1) \Leftrightarrow(2)$ follows immediately from [15], Theorem 0.8. This completes the proof of Proposition A.7.

Proposition A.8. Suppose that $p \notin \Sigma$, that $X$ admits good reduction over $\mathfrak{o}_{k}$, and that $X$ is proper over $k$. Then any pro- $\Sigma$ Galois section of $X / k$ [cf. [6], Definition 1.1, (i)] is unramified [cf. Definition A.1].

Proof. This follows immediately from the equivalence $(1) \Leftrightarrow(2)$ of Proposition A.6.

Proposition A.9. Suppose that $p \notin \Sigma$, and that $X$ admits good reduction over $\mathfrak{o}_{k}$. Then $s$ is unramified if and only if $s$ is potentially unramified [cf. Definition A.1].

Proof. This follows immediately from the equivalence $(1) \Leftrightarrow(2)$ of Proposition A.6, together with the well-known fact that any cuspidal inertia subgroup of $\Pi_{X / k}^{\Sigma-e ́ t}$ associated to a cusp of $X / k$ is isomorphic to $\widehat{\mathbb{Z}}^{\Sigma}$ as an abstract profinite group.

Proposition A.10. Let $Y$ be a hyperbolic curve over $k$ and $X \rightarrow Y a$ dominant morphism over $k$. Write $s_{Y}$ for the pro- $\Sigma$ Galois section of $Y / k$ [cf. [6], Definition 1.1, (i)] determined by s, i.e., the composite

$$
G_{k} \stackrel{s}{\longrightarrow} \Pi_{X / k}^{\Sigma} \longrightarrow \Pi_{Y / k}^{\Sigma}
$$

Then the following hold:

(i) Write $\Phi_{X / k}^{\Sigma}, \Phi_{Y / k}^{\Sigma}$ for the respective quotients of $\Pi_{X / k}^{\Sigma}, \Pi_{Y / k}^{\Sigma}$ defined in [7], Definition 1, (iv) [cf. also the statement of condition (3) of Proposition A.6]. Then the natural homomorphism $\Pi_{X / k}^{\Sigma} \rightarrow \Pi_{Y / k}^{\Sigma}$ induces a homomorphism $\Phi_{X / k}^{\Sigma} \rightarrow \Phi_{Y / k}^{\Sigma}$.

(ii) If $s$ is unramified (respectively, potentially unramified) [cf. Definition A.1], then $s_{Y}$ is unramified (respectively, potentially unramified).

(iii) Suppose that $X \rightarrow Y$ is finite, and that $X$ and $Y$ admit good reduction over $\mathfrak{o}_{k}$. Then $s$ is unramified if and only if $s_{Y}$ is unramified.

Proof. First, we verify assertion (i). Now since, as is well-known, the profinite group $\Delta_{Y / k}^{\Sigma}$ is slim [where we refer to the discussion entitled "Profinite groups" in $\S 0$ concerning the term "slim"], for any open subgroup $H \subseteq \Delta_{Y / k}^{\Sigma}$ of $\Delta_{Y / k}^{\Sigma}$, it follows immediately from [7], Lemma 5, that $N_{\Pi_{Y / k}^{\Sigma}}(H) \cap Z_{\Pi_{Y / k}^{\Sigma}}\left(\Delta_{Y / k}^{\Sigma}\right)=Z_{\Pi_{Y / k}^{\Sigma}}(H)$. Thus, it follows immediately from the fact that the natural homomorphism 
$\Pi_{X / k}^{\Sigma} \rightarrow \Pi_{Y / k}^{\Sigma}$ is open that the natural homomorphism $\Pi_{X / k}^{\Sigma} \rightarrow \Pi_{Y / k}^{\Sigma}$ induces a homomorphism $\Phi_{X / k}^{\Sigma} \rightarrow \Phi_{Y / k}^{\Sigma}$. This completes the proof of assertion (i). Assertion (ii) follows immediately from the various definitions involved, together with assertion (i) [cf. also the definitions of the quotients $\Phi_{X / k^{\prime}}^{\Sigma} \Phi_{Y / k}^{\Sigma}$. Finally, we verify assertion (iii). It follows from Proposition A.2, (i), that both the condition that $s$ is unramified and the condition that $s_{Y}$ is unramified imply that $p \notin \Sigma$. Thus, suppose that $p \notin \Sigma$. On the other hand, since $X \rightarrow Y$ is finite, one verifies easily that the restriction of the natural homomorphism $\Delta_{X / k}^{\Sigma} \rightarrow \Delta_{Y / k}^{\Sigma}$ to any cuspidal inertia subgroup of $\Pi_{X / k}^{\Sigma}$ associated to a cusp of $X / k$ is injective. Thus, assertion (iii) follows immediately from assertions (i), (ii); the equivalence (1) $\Leftrightarrow$ (3) of Proposition A.6, together with the fact that the sequences of natural surjections $\Pi_{X / k}^{\Sigma} \rightarrow \Pi_{X / k}^{\Sigma-\text { ét }} \rightarrow \Phi_{X / k}^{\Sigma}, \Pi_{Y / k}^{\Sigma} \rightarrow \Pi_{Y / k}^{\Sigma \text {-ét }} \rightarrow \Phi_{Y / k}^{\Sigma}$ induce injections $\Delta_{X / k}^{\Sigma} \stackrel{\sim}{\rightarrow} \Delta_{X / k}^{\Sigma-e ́ t} \hookrightarrow \Phi_{X / k}^{\Sigma}, \Delta_{Y / k}^{\Sigma} \stackrel{\sim}{\rightarrow} \Delta_{Y / k}^{\Sigma \text {-ét }} \hookrightarrow \Phi_{Y / k}^{\Sigma}$ respectively [cf. the proof of the implication (2) $\Rightarrow$ (3) of Proposition A.6]. This completes the proof of assertion (iii).

\section{REFERENCES}

[1] H. Esnault and O. Wittenberg, On abelian birational sections, J. Amer. Math. Soc. 23 (2010), no. 3, 713-724.

[2] J.-H.Evertse, H. P. Schlickewei, and W. M. Schmidt, Linear equations in variables which lie in a multiplicative group, Ann. of Math. (2) 155 (2002), no. 3, 807-836.

[3] A. Grothendieck et al, Revêtements étales et groupe fondamental (SGA 1), Séminaire de géométrie algébrique du Bois Marie 1960-61, Documents Mathématiques, 3. Société Mathématique de France, Paris, 2003.

[4] D. Harari and J. Stix, Descent obstruction and fundamental exact sequence, The Arithmetic of Fundamental Groups - PIA 2010, 147-166, Contributions in Mathematical and Computational Sciences, vol. 2, Springer-Verlag Berlin Heidelberg, 2012.

[5] Y. Hoshi and S. Mochizuki, On the combinatorial anabelian geometry of nodally nondegenerate outer representations, Hiroshima Math. J. 41 (2011), no. 3, 275-342.

[6] Y. Hoshi, Existence of nongeometric pro- $p$ Galois sections of hyperbolic curves, Publ. Res. Inst. Math. Sci. 46 (2010), no. 4, 829-848.

[7] Y. Hoshi, On monodromically full points of configuration spaces of hyperbolic curves, The Arithmetic of Fundamental Groups - PIA 2010, 167-207, Contributions in Mathematical and Computational Sciences, vol. 2, Springer-Verlag Berlin Heidelberg, 2012.

[8] J. Koenigsmann, On the 'section conjecture' in anabelian geometry, J. Reine Angew. Math. 588 (2005), 221-235.

[9] S. Mochizuki, The local pro- $p$ anabelian geometry of curves, Invent. Math. 138 (1999), no. 2, 319-423.

[10] S. Mochizuki, Topics surrounding the anabelian geometry of hyperbolic curves, Galois groups and fundamental groups, 119-165, Math. Sci. Res. Inst. Publ. 41, Cambridge Univ. Press, Cambridge, 2003. 
[11] D. Mumford, Abelian varieties, Tata Institute of Fundamental Research Studies in Mathematics, No. 5, Published for the Tata Institute of Fundamental Research, Bombay; Oxford University Press, London 1970.

[12] F. Pop, On the birational p-adic section conjecture, Compos. Math. 146 (2010), no. 3, 621-637.

[13] J.-P. Serre and J. Tate, Good reduction of abelian varieties, Ann. of Math. (2) 88 1968 492-517.

[14] M. Stoll, Finite descent obstructions and rational points on curves, Algebra Number Theory 1 (2007), no. 4, 349-391.

[15] A. Tamagawa, The Grothendieck conjecture for affine curves, Compositio Math. 109 (1997), no. 2, 135-194.

[16] K. Wickelgren, 2-nilpotent real section conjecture, arXiv:1006.0265, 2010.

(Yuichiro Hoshi) Research Institute for Mathematical Sciences, KYOTO UNIVERSITY, KYOTO 606-8502, JAPAN

E-mail address: yuichiro@kurims.kyoto-u.ac.jp 